\title{
Non-cross Bridge Viscoelastic Elements Contribute to Muscle Force and Work During Stretch-Shortening Cycles: Evidence From Whole Muscles and Permeabilized Fibers
}

\author{
Anthony L. Hessel ${ }^{1 \dagger}$, Jenna A. Monroy ${ }^{2 * t}$ and Kiisa C. Nishikawa ${ }^{3}$ \\ 1/nstitute of Physiology II, University of Muenster, Muenster, Germany, ${ }^{2}$ W.M. Keck Science Department, Claremont Colleges, \\ Claremont, CA, United States, ${ }^{3}$ Department of Biological Sciences, Northern Arizona University, Flagstaff, AZ, United States
}

\section{OPEN ACCESS}

Edited by:

Geoffrey A. Power,

University of Guelph, Canada

Reviewed by:

Arthur J. Cheng,

York University, Canada

Atsuki Fukutani,

University of Calgary, Canada

${ }^{*}$ Correspondence:

Jenna A. Monroy

JMonroy@kecksci.claremont.edu

these authors have contributed equally to this work and share first

authorship

Specialty section: This article was submitted to Striated Muscle Physiology, a section of the journal

Frontiers in Physiology

Received: 31 December 2020 Accepted: 08 March 2021

Published: 29 March 2021

Citation:

Hessel AL, Monroy JA and Nishikawa KC (2021) Non-cross

Bridge Viscoelastic Elements

Contribute to Muscle Force and Work

During Stretch-Shortening Cycles:

Evidence From Whole Muscles and Permeabilized Fibers.

Front. Physiol. 12:648019.

doi: 10.3389/fphys.2021.648019
The sliding filament-swinging cross bridge theory of skeletal muscle contraction provides a reasonable description of muscle properties during isometric contractions at or near maximum isometric force. However, it fails to predict muscle force during dynamic length changes, implying that the model is not complete. Mounting evidence suggests that, along with cross bridges, a $\mathrm{Ca}^{2+}$-sensitive viscoelastic element, likely the titin protein, contributes to muscle force and work. The purpose of this study was to develop a multi-level approach deploying stretch-shortening cycles (SSCs) to test the hypothesis that, along with cross bridges, $\mathrm{Ca}^{2+}$ sensitive viscoelastic elements in sarcomeres contribute to force and work. Using whole soleus muscles from wild type and $m d m$ mice, which carry a small deletion in the N2A region of titin, we measured the activation- and phase-dependence of enhanced force and work during SSCs with and without doublet stimuli. In wild type muscles, a doublet stimulus led to an increase in peak force and work per cycle, with the largest effects occurring for stimulation during the lengthening phase of SSCs. In contrast, $m d m$ muscles showed neither doublet potentiation features, nor phase-dependence of activation. To further distinguish the contributions of cross bridge and non-cross bridge elements, we performed SSCs on permeabilized psoas fiber bundles activated to different levels using either [ $\left.\mathrm{Ca}^{2+}\right]$ or $\left[\mathrm{Ca}^{2+}\right]$ plus the myosin inhibitor 2,3-butanedione monoxime (BDM). Across activation levels ranging from 15 to $100 \%$ of maximum isometric force, peak force, and work per cycle were enhanced for fibers in $\left[\mathrm{Ca}^{2+}\right]$ plus BDM compared to $\left[\mathrm{Ca}^{2+}\right]$ alone at a corresponding activation level, suggesting a contribution from $\mathrm{Ca}^{2+}$-sensitive, non-cross bridge, viscoelastic elements. Taken together, our results suggest that a tunable viscoelastic element such as titin contributes to: (1) persistence of force at low [ $\left.\mathrm{Ca}^{2+}\right]$ in doublet potentiation; (2) phase- and length-dependence of doublet potentiation observed in wild type muscles and the absence of these effects in mdm muscles; and (3) increased peak force and work per cycle in SSCs. We conclude that non-cross bridge viscoelastic elements, likely titin, contribute substantially to muscle force and work, as well as the phase-dependence of these quantities, during dynamic length changes.

Keywords: 2,3-butanedione monoxime, calcium-dependence, doublet potentiation, skeletal muscle, titin, work loop, history-dependence 


\section{INTRODUCTION}

As scientific theories evolve, paradigms often shift as seemingly obscure facts that resisted explanation by an accepted theory take on new importance (Kuhn, 1962). In the sliding-filament, swinging cross bridge theory of muscle contraction (Huxley and Hanson, 1954; Huxley and Niedergerke, 1954; Huxley, 1957), the isometric force-length relationship (Gordon et al., 1966), and the isotonic force-velocity relationship (Huxley, 1957) played important roles in establishing the theory as fact. However, several key observations including enhancement of force with stretch and depression of force with shortening resisted explanation by the accepted theory (Abbott and Aubert, 1952; Edman, 1975; Edman et al., 1982; Tsuchiya and Sugi, 1988). The perceived importance of these history-dependent muscle properties has increased over recent decades with the recognition that they play essential roles in human and animal movement by enhancing work and power output, dissipating force, and providing instantaneous stabilization during unexpected perturbations (Daley and Biewener, 2011; Nishikawa et al., 2013; Seiberl et al., 2013). Additionally, there is increasing recognition that muscle models based on the isometric forcelength and isotonic force-velocity relationships fail to predict muscle force during dynamic in vivo movement (Siebert et al., 2008; Lee et al., 2013; Dick et al., 2017), perhaps because a critical element is missing (Rode et al., 2009; Heidlauf et al., 2017; Nishikawa et al., 2018; Nishikawa, 2020).

Accumulating evidence suggests that $\mathrm{Ca}^{2+}$-sensitive, non-cross bridge, viscoelastic elements in sarcomeres of skeletal muscles (Monroy et al., 2012; Colombini et al., 2016; Nishikawa et al., 2019), are responsible for history-dependent muscle properties during changes in length (Nocella et al., 2014; Nishikawa, 2016; Herzog, 2018). It has also become increasingly accepted that the giant titin protein is a $\mathrm{Ca}^{2+}$-sensitive, viscoelastic element in muscle sarcomeres (Nishikawa, 2020). Titin is the largest known protein, extending from the Z-line to the M-band (Bang et al., 2001; Linke, 2018). In the Z-line and A-band, titin is bound to the thin and thick filaments, respectively. In contrast, I-band titin is a freely extensible, viscoelastic spring, that is predominantly comprised of a relatively compliant proximal Ig domain region, a stiff PEVK region, (named for its predominant residues), and an $\mathrm{N} 2 \mathrm{~A}$ region that spans between the two springs (Linke et al., 1998a,b). Because of its location within the sarcomere, titin is responsible for nearly all longitudinal force in relaxed myofibrils (Granzier and Irving, 1995) and was suggested to function as a $\mathrm{Ca}^{2+}$-dependent spring in active muscle (Leonard and Herzog, 2010; Nishikawa et al., 2012; Linke, 2018). Recent work has demonstrated that the N2A region of titin binds to actin in a $\mathrm{Ca}^{2+}$-dependent manner (Dutta et al., 2018; Nishikawa et al., 2019), which would shorten titin's free length and allow for stretch of only the stiffer PEVK region in active muscle. The $m d m$ mutation in mice produces a small deletion in N2A titin (Garvey et al., 2002), which profoundly reduces active muscle stiffness (Powers et al., 2016; Hessel and Nishikawa, 2017; Monroy et al., 2017), leading to a reduction in both force enhancement with stretch and force depression with shortening
(Tahir et al., 2020), apparently by preventing N2A titin-actin binding (Dutta et al., 2018; Nishikawa et al., 2019).

Many previous experiments have evaluated force during isovelocity stretch-hold (i.e., force enhancement) or shortenhold (i.e., force depression) contractions (recently reviewed by Herzog, 2018; Chen et al., 2019). While these experiments provide important details about the history-dependence of force during and after isovelocity stretch and shortening, they fail to capture the history-dependent properties of muscles during SSCs (Seiberl et al., 2015b; Hahn and Riedel, 2018; Fukutani and Herzog, 2020b), in which energy stored during stretch can be recovered during shortening to increase net work per cycle (Hahn and Riedel, 2018; Fukutani and Herzog, 2020b). Most previous ex vivo studies using SSCs typically varied the velocity or amplitude of length changes (Askew and Marsh, 1997, 1998), but only rarely have such studies examined the effects of cross-bridge inhibition on muscle force and work (Fukutani and Herzog, 2020b). While it is now widely believed that cross bridges contribute relatively little to energy storage during active stretch (Linari et al., 2003; Pinniger et al., 2006), the mechanisms and extent of contributions from non-cross bridge elements such as titin to energy storage and recovery in SSCs remains to be elucidated. Based on the above arguments, it seems reasonable that a $\mathrm{Ca}^{2+}$-activated, non-cross bridge, viscoelastic element, specifically titin, contributes to muscle force and work during SSCs.

In this study, we used a multi-level, ex vivo approach to test broadly for contributions of $\mathrm{Ca}^{2+}$-activated, non-cross bridge, viscoelastic elements to SSCs in two different experimental preparations and muscles: whole soleus muscles and permeabilized psoas fibers. In whole muscle experiments, we used doublet stimuli at different phases of SSCs in wild type and $m d m$ muscles to test for a role of titin in activation-and phase-dependence of peak force and work. In permeabilized psoas fibers, we used the myosin inhibitor, BDM to investigate contributions of $\mathrm{Ca}^{2+}$-sensitive non-cross bridge elements at different activation levels. By taking similar approaches that vary activation in the two preparations, we aim to further our understanding of the role of titin in regulating muscle force and work during SSCs.

For whole muscles, our strategy was to use doublet stimuli at varying phases of SSCs in wild type and $m d m$ soleus muscles to test whether titin contributes not only to muscle force and work, but also to doublet potentiation as well as to the phasedependence of activation, which feature prominently in the biomechanics of in vivo movements (Dickinson et al., 2000; Seiberl et al., 2015a; Paternoster et al., 2016). Doublet stimuli, when added to a train of low-frequency stimuli, potentiate muscle force (Burke et al., 1970, 1976) for up to hundreds of milliseconds after the single extra stimulus (Sandercock and Heckman, 1997). Although few previous studies have investigated doublet potentiation in SSCs, a single stimulus has been observed to increase work per cycle by up to $50 \%$ (Stevens, 1996). While this property of muscle has been known for more than 50 years, the underlying mechanisms are not well explained by the sliding filament-swinging cross bridge theory of muscle contraction (Burke et al., 1970; Sandercock and Heckman, 1997; 
Binder-Macleod and Kesar, 2005; Nishikawa et al., 2018). Several studies have shown that the $\mathrm{Ca}^{2+}$ transient associated with the doublet stimulus returns to control levels within $\sim 25 \mathrm{~ms}$ (Abbate et al., 2002; Cheng et al., 2013; Bakker et al., 2017), but the increase in muscle force that persists for hundreds of milliseconds cannot be explained by current theories (Burke et al., 1970; Binder-Macleod and Kesar, 2005; Nishikawa, 2018). Previous studies have suggested that, similar to invertebrate "catch" (Butler and Siegman, 2010), engagement of a $\mathrm{Ca}^{2+}$-activated elastic element could potentially explain the sustained force at low $\left[\mathrm{Ca}^{2+}\right]$ following doublet stimulation (Parmiggiani and Stein, 1981; Sandercock and Heckman, 1997; Binder-Macleod and Kesar, 2005; Nishikawa, 2018). By varying the phase of doublet stimulation in SSCs, we also investigated a potential role for titin in the phasedependence of activation (Dickinson et al., 2000; Ahn, 2012).

For permeabilized muscle fibers, we developed a different strategy to investigate the relative contributions of $\mathrm{Ca}^{2+}$-sensitive cross bridges and non-cross bridge elements to muscle force and work in SSCs. Many previous studies have reported that, as activation increases in SSCs, peak force during lengthening also increases, which correlates with increased positive work during the subsequent shortening phase (Seiberl et al., 2015b; Fortuna et al., 2017; Fukutani and Herzog, 2020a). Of note, treatment of single muscle fibers with $10 \mathrm{mM}$ BDM ( 50\% maximum activation level) enhanced shortening work during SSCs relative to a shortening-only contraction (Fukutani and Herzog, 2020b), suggesting that a $\mathrm{Ca}^{2+}$-sensitive, non-cross bridge viscoelastic element remains engaged during myosin inhibition. Here, we extend these observations to a wide range of concentration of calcium ions ( $\mathrm{pCa}$ )- and BDM-regulated activation levels (15-100\%). If a $\mathrm{Ca}^{2+}$-sensitive, non-cross bridge viscoelastic element remains engaged during myosin inhibition, then we expect that peak force and work during SSCs will be greater in BDM-treated than in $\mathrm{Ca}^{2+}$-activated fiber bundles matched to the same level of activation. We measured force and work of permeabilized fiber bundles during SSCs across differing levels of activation, achieved by varying the $\mathrm{pCa}$ and the myosin inhibitor, 2,3-butanedione monoxime (BDM) in a series of activating solutions. BDM reduces the proportion of actin-bound myosin heads (Griffiths et al., 2006), and therefore the isometric force of $\mathrm{Ca}^{2+}$-activated fibers. We matched activation levels of mouse psoas fiber bundles activated using solutions with different $\mathrm{pCa}$ to similar bundles activated using varying doses of $\mathrm{BDM}$ at $\mathrm{pCa}=4.2$. By comparing BDM-controlled and pCa-controlled fibers at a given activation level, we sought to determine whether force and work during SSCs were produced by cross bridges alone, or whether there was evidence for a contribution of additional $\mathrm{Ca}^{2+}$-dependent, viscoelastic elements.

In summary, our multi-level approach focuses on varying activation levels in SSCs: using $\mathrm{pCa}$ and BDM in psoas fiber bundles, and changing stimulation patterns (i.e., doublets and stimulation phase) in whole soleus muscles. At the whole muscle level, we hypothesize that titin contributes to doublet potentiation of muscle force and work during SSCs, and that the $m d m$ mutation reduces not only the doublet effect but also the phase-dependence of activation on muscle force and work. At the level of permeabilized fibers, we predicted that, if only cross bridges contribute to force and work, and then there should be no difference in SSCs between BDM-controlled vs. pCa-controlled fibers across activation levels. However, if $\mathrm{Ca}^{2+}$-dependent viscoelastic elements also contribute, then BDM-controlled fibers should show greater force and work than pCa-controlled fibers.

\section{MATERIALS AND METHODS}

Animal use was approved by the Institutional Animal Care and Use Committees of the University Clinic Muenster (LANUV NRW, 81-02.04.2019.A472), Northern Arizona University (NAU IACUC Protocol \#18-002), and the Claremont Colleges (CC IACUC Protocol \#017-003). Adult mice were euthanized by an isoflurane gas overdose, cervical dislocation, and cardiac puncture. For whole muscle studies, wild type and homozygous recessive $(m d m)$ B6C3Fe a/a-Ttn ${ }^{m d m} / J$ mice from a C57BL/6 J background were obtained from a breeding colony at Northern Arizona University. Homozygous $m d m$ mice can be identified by their small size, stiff gait, and hunchback posture at 10-12 days of age (Garvey et al., 2002; Lopez et al., 2008). Fiber studies were conducted on wild type mice with the same genetic background (C57BL/6 J) from a colony at University of Muenster.

\section{Whole Muscle Experiments}

Whole muscle experiments were conducted on 11 wild type and $18 \mathrm{mdm}$ soleus muscles from age-matched mice of both sexes (average wild type age $=44.4 \pm 2.0$ days; average $m d m$ age $=49.6 \pm 2.9$ days, $p=0.12$ ). Because wild type mice were larger in body size than $m d m$ mice, muscle mass, optimal length $\left(\mathrm{L}_{0}\right)$, and maximum isometric force were significantly greater in wild type compared to $m d m$ soleus (Table 1), as reported previously (Taylor-Burt et al., 2015; Hessel and Nishikawa, 2017; Monroy et al., 2017; Hessel et al., 2019; Tahir et al., 2020). Maximum isometric stress was also lower in $m d m$ muscles, likely because titin transmits cross bridges forces from the A-band to the Z-disk (Horowits et al., 1986; Nishikawa, 2020). Neither $\mathrm{Ca}^{2+}$ sensitivity nor the force-velocity relationship differs between genotypes (Hessel and Nishikawa, 2017; Tahir et al., 2020). To account for differences in length and stress, muscle length was normalized to optimal length $\left(\mathrm{L}_{0}\right)$ and relative differences in stress were compared between genotypes.

To obtain force and length measurements during SSCs, the distal end of each muscle was attached to an inflexible hook,

TABLE 1 | Physiological characteristics of wild type and mdm soleus muscles.

\begin{tabular}{lcr}
\hline & $\boldsymbol{m d \boldsymbol { m }}$ & Wild type \\
\hline Body mass $(\mathrm{g})$ & $7.5 \pm 0.30^{*}$ & $23.4 \pm 0.49$ \\
Muscle mass $(\mathrm{mg})$ & $1.96 \pm 0.53^{*}$ & $9.3 \pm 0.67$ \\
$\mathrm{~L}_{0}(\mathrm{~mm})$ & $7.13 \pm 0.26^{*}$ & $11.27 \pm 0.33$ \\
Max isometric force $(\mathrm{N})$ & $0.008 \pm 0.006^{*}$ & $0.14 \pm 0.01$ \\
ForForcewFocforce $(\mathrm{N})$ & $4.1 \pm 1.2^{*}$ & $15.6 \pm 1.53$ \\
$\mathrm{P}_{0}\left(\mathrm{Ncm}^{-2}\right)$ & &
\end{tabular}

Data expressed as mean \pm SEM, mam: $n=18$, wild type: $n=11 .{ }^{*}$ indicates $p<0.05$ 
and the proximal end was attached to a dual servomotor muscle lever (Aurora Scientific, Inc., Series 300B, Aurora, ON, Canada). A custom LabVIEW (National Instruments Corp., Austin, TX, United States) program was used to control the muscle lever and record force, length and time at a sampling rate of $4 \mathrm{kHz}$. Muscles were kept in a Krebs-Henseleit bath (in mM: 137 $\mathrm{NaCl}, 5 \mathrm{KCl}, 1 \mathrm{NaH}_{2} \mathrm{PO}_{4}, 24 \mathrm{NaHCO}_{3}, 2 \mathrm{CaCl}_{2}, 1 \mathrm{MgSO}_{4}$, and 11 dextrose, $\mathrm{pH} 7.4$ ), buffered with $95 \% \mathrm{O}_{2}$ and $5 \% \mathrm{CO}_{2}$. Because SSC experiments can fatigue muscles quickly, whole muscles were maintained at a constant temperature $\left(21-23^{\circ} \mathrm{C}\right)$, at which the maximum isometric tetanic force remains stable for several hours and within $10 \%$ of the maximum isometric stress at normal body temperature of $37^{\circ} \mathrm{C}$ (James et al., 2015).

Maximum isometric tetanic force was measured from soleus muscles stimulated at $70-80 \mathrm{~Hz}$ for $800-1,500 \mathrm{~ms}$ at supramaximal voltage (Monroy et al., 2017). Periodically, the maximum isometric tetanic force was measured, and a muscle was removed from the analysis if force dropped by more than $10 \%$ from the initial value. At the start of each SSC experiment, muscles were set to optimal length $\left(\mathrm{L}_{0}\right)$, defined as the length at which maximum isometric twitch force was produced. The maximum isometric stress $\left(\mathrm{P}_{0}, \mathrm{Ncm}^{-2}\right)$ was determined by dividing maximum isometric force by the physiological cross-sectional area, calculated using standard methods (Hakim et al., 2013; Monroy et al., 2017).

We measured the effects of doublet potentiation at different phases during SSCs in wild type and $m d m$ soleus muscles to determine not only whether titin contributes to muscle force and work, but also to the activation- and phase-dependence of these quantities. Small amounts of doublet potentiation in fast-twitch muscles stimulated at nearly the maximum frequency can be explained by an increased number of cross bridges (Bakker et al., 2017), but not the larger and longer lasting effects that are observed at lower stimulation frequencies (see e.g., Abbate et al., 2002). Long-lasting doublet potentiation in soleus muscles that persists for $>200 \mathrm{~ms}$ also is not readily explained by cross-bridge models (Sandercock and Heckman, 1997). By measuring muscle work during SSCs in wild type and $m d m$ soleus muscles with and without doublet stimulation, we can determine whether titin likely contributes to doublet potentiation of muscle force and work. Muscles were subjected to $\pm 5 \% \mathrm{~L}_{0}$ SSCs at a frequency of $5 \mathrm{~Hz}$ for a total of four cycles with and without doublet stimulation (Figures 1A,C). The cycle phase is defined here as the percent of an individual stretch-shortening cycle (SSC), where a phase of $0 \%$ denotes a muscle at its shortest length $\left(-5 \% \mathrm{~L}_{0}\right)$ at the onset of lengthening, a phase of $50 \%$ denotes a muscle at its longest length $\left(+5 \% \mathrm{~L}_{0}\right)$ at the onset of shortening, and the cycle ends at a phase of $100 \%$ (Figures 1B,D). Muscles were stimulated submaximally at $30 \mathrm{~Hz}$ for $100 \mathrm{~ms}$.

To investigate the phase dependence of doublet stimulation, activation was initiated at six different phases within the SSC $(8.3,25,33.3,50$, and $83.3 \%)$, which determined muscle length at the onset of stimulation as well as the direction of the work loop (clockwise for positive work and counter-clockwise for negative work). Each activation onset was repeated with and without a single doublet stimulus. Doublet stimuli were added $10 \mathrm{~ms}$ following the initial stimulus, for a total of three or four stimuli per cycle (see Figure 1; doublet stimulus indicated by black arrow). Passive and maximally stimulated (i.e., $75-80 \mathrm{~Hz}$ stimulus frequency) work loops were also obtained at each phase of stimulation. Muscles were rested 5-7 min between trials to minimize fatigue. For each trial, peak stress $\left(\mathrm{Ncm}^{-2}\right)$ and mass-specific net, negative, and positive work were measured from the third cycle of each trial (James et al., 1995) using MATLAB (2019) [version 9.7.0. (R2019b) Natick, Massachusetts: The MathWorks Inc.].

\section{Permeabilized Fiber Bundle Experiments}

Permeabilized ("skinned") fibers were prepared from wild type mouse psoas muscles ( $n=8$ mice, five male/three female, age range 3-6 months) using standard glycerol techniques (Hessel et al., 2019). Extracted muscles were permeabilized and stored in a relaxing solution [in $\mathrm{mmol}^{-1}$ : potassium propionate (170), magnesium acetate (2.5), MOPS (20), $\mathrm{K}_{2}$ EGTA (5), and ATP (2.5), $\mathrm{pH}$ 7.0] for $12 \mathrm{~h}$ at $4^{\circ} \mathrm{C}$, then moved to a relaxing: glycerol (50:50) or rigor: glycerol solution [ $\mathrm{KCl}(100), \mathrm{MgCl}_{2}$ (2), EGTA (5), Tris (10), DTT (1), 50\% glycerol, and pH 7.0] at $-20^{\circ} \mathrm{C}$ for a minimum of 3 weeks. To limit protein degradation, all solutions contained one tablet of protease inhibitor (Complete, Roche Diagnostics, Mannheim, Germany) per $100 \mathrm{ml}$ of solution. On the day of experiments, permeabilized muscles were removed from the storage solution and vigorously washed in relaxing solution on ice. Small bundles of 3-10 fibers were separated from the muscle. The ends of the fibers were wrapped with fine sutures and placed in custom-made clips to secure the fibers in place. Fibers bundles were attached lengthwise to a piezomotor on one end and a force transducer on the other end via aluminum clamps (Scientific Instruments, Heidelberg, Germany). All mechanical experiments were performed at $21^{\circ} \mathrm{C}$. Force data were recorded at 1,000 $\mathrm{Hz}$. Each fiber bundle was suspended in a bath of relaxing solution and then transferred to other baths as needed. Sarcomere length (SL) was measured by laser diffraction and initially set to an average length of $2.6 \mu \mathrm{m}$ SL. Length changes were accomplished by computercontrol of the length motor. Force was set to slack length $=0 \mathrm{mN}$ for each sample. Total force is a combination of passive and active force components.

To separate the effects of $\left[\mathrm{Ca}^{2+}\right]$ on cross bridge and non-cross bridge elements, we conducted mechanical experiments on fiber bundles activated to different levels by either: (1) changing the level of $\left[\mathrm{Ca}^{2+}\right]$ (expressed as $\mathrm{pCa}=-\log \left[\mathrm{Ca}^{2+}\right]$ ); or (2) reducing cross bridge force production using the myosin inhibitor $\mathrm{BDM}$ at supramaximal $\mathrm{pCa}=4.2$. Different activating solutions were prepared from the same stock activating solution [in mmol $1^{-1}$ : potassium propionate (170), magnesium acetate $(2.5)$, MOPS (10), ATP (2.5), and pH 7.0] by adding CaEGTA and $\mathrm{K}_{2}$ EGTA in different proportions to obtain different pCa levels. For pCa-controlled experiments, we prepared activating solutions with a final pCa range between 4.2 and 6.2 to achieve a range of isometric forces (confirmed during pilot tests). For the BDM-controlled solutions, we added BDM to supramaximal activation solutions ( $\mathrm{pCa} 4.2$ ) to final concentrations between 
A

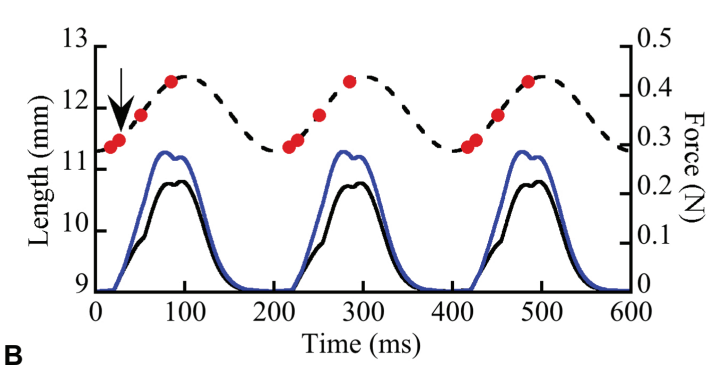

B

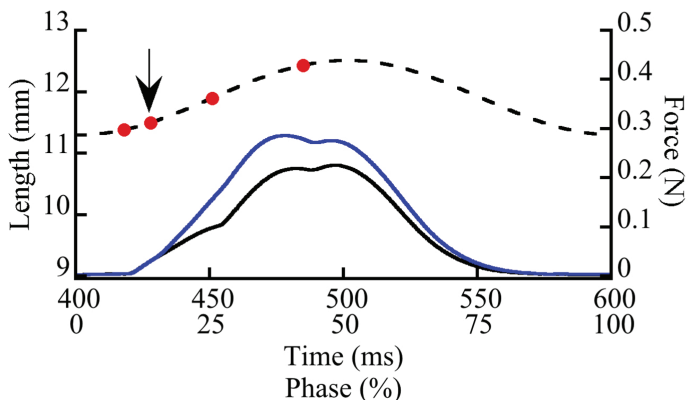

C
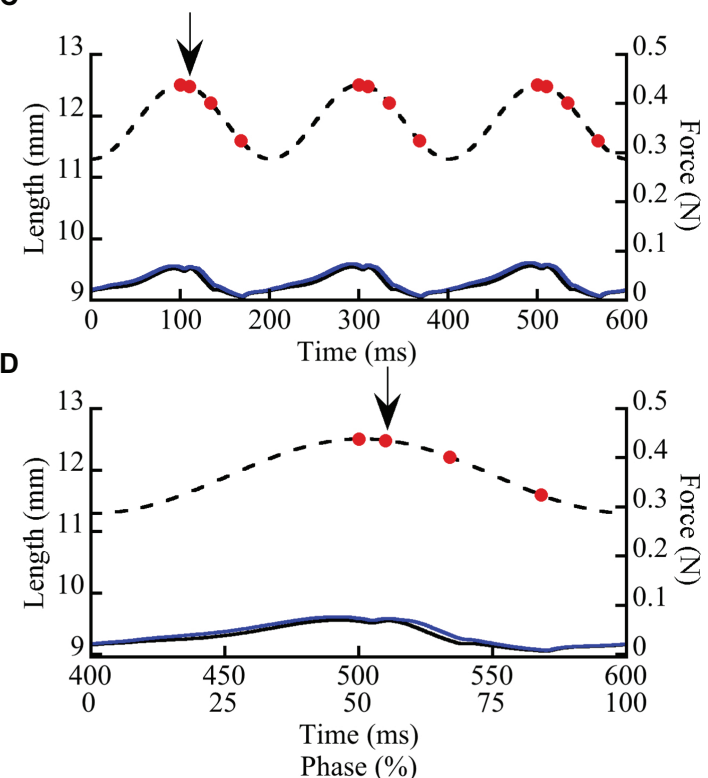

FIGURE 1 | Representative stretch-shortening cycles (SSCs) from whole wild type soleus muscles stimulated at two phases of activation (8.3 and 50\%). Muscles were subjected to 3-4 SSCs at strain amplitude of $\pm 5 \% \mathrm{~L}_{0}$ and a frequency of $5 \mathrm{~Hz}$ (200 ms/cycle). In this example, a muscle was stimulated at a phase of $8.3 \%$ (A,B) when the muscle was lengthening and at a phase of $50 \%$ (C,D) at the onset of muscle shortening. (A,C) illustrate three successive cycles. (B,D) show the third cycle from each trial, as well as the relationship between time (ms) and phase (0-100\%). Dashed lines show length vs. time and solid lines show force vs. time. Muscle force (solid lines) is shown for SSCs with (blue lines) and without (black lines) a doublet stimulus added 10 ms after the first stimulus in each cycle. Red dots indicate individual stimuli, the arrow indicates the doublet.

0 and $30 \mathrm{mM} \mathrm{BDM}$, to achieve a range of isometric forces from 100 to $\sim 15 \%$ of maximum isometric force. The goal was to perform SSCs on individual fiber bundles over as a wide range of activation levels as possible to reduce variability associated with comparing data across different samples. Fibers were subjected to either the BDM- or the $\mathrm{pCa}$ - controlled protocols, but not both; we found that some level of forcereduction remained after BDM treatment, even with thorough washing.

The SSC protocol was as follows (Figure 2): muscle fibers were either kept relaxed or isometrically activated at $2.6 \mu \mathrm{m}$ SL until isometric force was reached (1.5-4 $\mathrm{min})$, and then cycled through eight successive triangular SSCs, from 2.6 to $3.0 \mu \mathrm{m} \mathrm{SL}$ at $2 \mathrm{~Hz}\left(1.6 \mu \mathrm{m} \mathrm{SL} \mathrm{s}{ }^{-1}\right)$. After completion of SSCs, fibers were held at constant length $(2.6 \mu \mathrm{m} \mathrm{SL})$ for $30 \mathrm{~s}$, and finally deactivated in relaxing solution. All fibers were tested in the following order: (1) Passive fibers were moved through the SSC protocol in a relaxed state 3-5 times to pre-stress the sample, which makes the force data more consistent ( $\mathrm{Li}$ et al., 2020; Rivas-Pardo et al., 2020). The final passive SSC protocol was saved and used as the relaxed state. (2) Fibers were maximally activated ( $\mathrm{pCa} 4.2$ ) and moved through the SSC protocol. (3) The SSC protocol was repeated from lowest to highest activating solutions (increasing activation level for both $\mathrm{BDM}$ and $\mathrm{pCa}$ trials), up to seven different activating solutions per fiber, with at least $3 \mathrm{~min}$ rest in fresh relaxing solution between trials. This order was followed to maximize the number of tests that could be performed on a single fiber bundle before the force it produced dropped below $85 \%$ of its original maximum force, after which the fiber bundle was no longer used in experiments. We chose to use seven trials per sample after an extensive pilot study in which we subjected fiber bundles to various levels of $\mathrm{pCa}$ and measured changes in force over time. At certain submaximal activation levels, we found that fiber bundles could be activated up to 20 times before forces dropped below $85 \%$ of starting values. The conservative seven-trial cutoff was chosen to ensure that most fibers would be able to complete the experiment with minimal decrease in force. For each fiber bundle, the activation level (\%) was calculated as the isometric force prior to the SSC divided by the maximum isometric force of the same fiber bundle at $\mathrm{pCa}=4.2$.

Generally, activation led to a typical leveling-off of isometric force over time, but the time to reach maximum force increased as activation level decreased (see Figures 2A,B). Some experiments at the lowest activation levels (>25 mM BDM; > pCa 6.1) required up to 4 min to develop maximum isometric force. Therefore, we used relatively long isometric activations before SSCs in all trials. After each trial, the fibers were visually assessed for damage using a 10x dissection microscope. We excluded the data and terminated the experiment if fibers tore, or if the passive force of relaxed fibers changed after the SSC protocol by $>5 \%$. In the present study, 12/43 fiber bundles were discarded. To compare across different fiber bundles, force data ( $\mathrm{mN}$ total force) were normalized to stress $\left(\mathrm{mN}^{\star} \mathrm{mm}^{2}\right)$ using standard methods (Hessel et al., 2019). Length data were 
A

\section{pCa-Controlled}

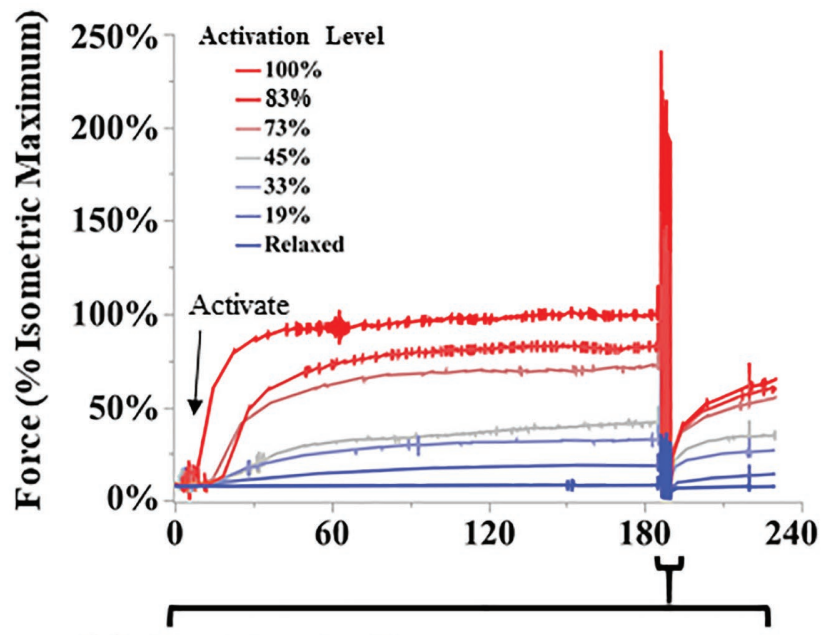

3.0- Length change (um SL)

2.4-

C

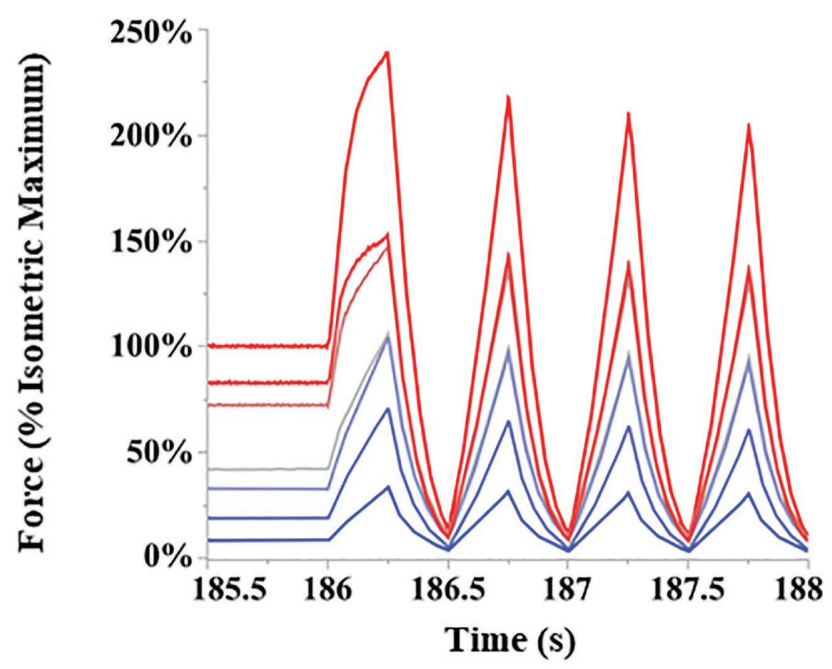

B

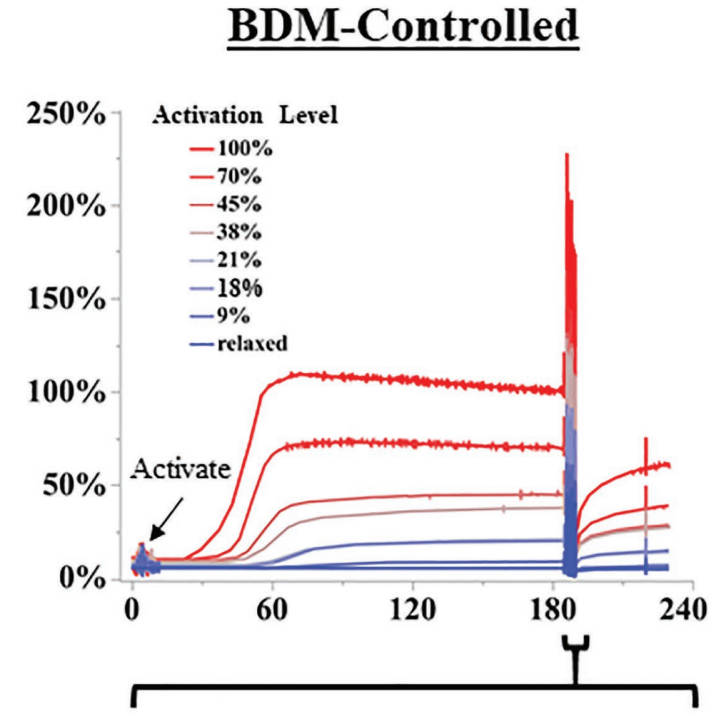

3.0- Length change (um SL)

2.4-

D

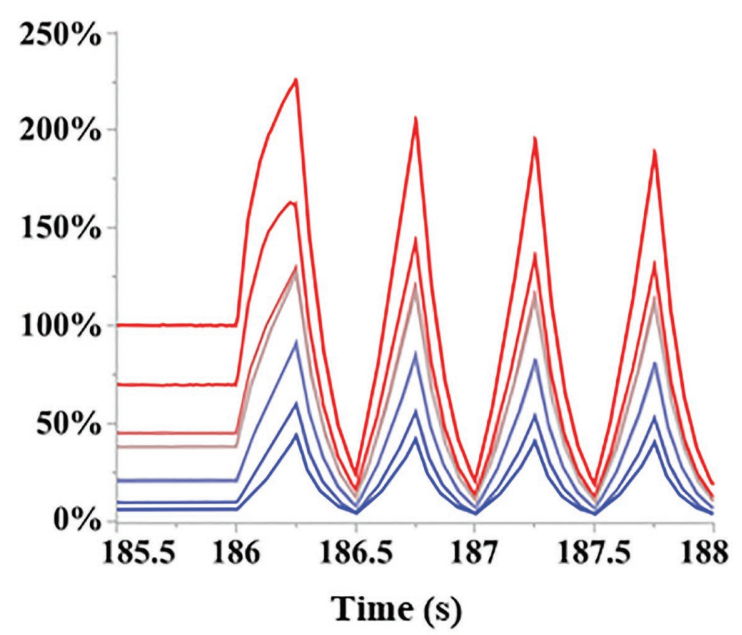

FIGURE 2 | Representative SSC experiments from permeabilized fiber bundles. Activation levels were controlled either by decreasing the concentration of calcium ions ( $\mathrm{pCa} ; \mathbf{A}, \mathbf{C})$, or by 2,3-butanedione monoxime (BDM)-induced myosin inhibition (B,D). Length changes were performed using a "triangular" length trajectory between 2.4 and $3.0 \mu \mathrm{m} \mathrm{SL}$ at $2 \mathrm{~Hz}$. (A,B) show all force trials collected for a representative fiber bundle for pCa-controlled and BDN-controlled fibers, respectively. Bundles were activated until force leveled off, and then subjected to multiple SSCs. (C,D) show the first four cycles at a higher time resolution for pCa and BDM trials, respectively.

normalized to strain using the initial length of $2.6 \mu \mathrm{m}$ SL. Peak force and specific work were normalized to the fiber's isometric force at pCa 4.2 (reference trial). In total, we collected contraction trials from 16 and 15 fiber bundles in BDM and pCa-controlled protocols, respectively.

\section{Statistical Analysis}

Statistical analyses were conducted using the lme4, lmtest, tidyverse, car (Fox and Weisberg, 2010), and MASS packages in R Studio statistical software (R Studio Team, 2020), Microsoft Excel (v11, Microsoft Inc., Seattle, WA, United States), and JMP (JMP Pro 14, SAS Institute). Alpha values were set at 0.05. Data were best Box-Cox transformed to meet assumptions of normality and homoscedasticity when necessary. Data are presented as mean $\pm \mathrm{SE}$ (sem).

\section{Whole Muscle Experiments}

To assess effects of genotype and phase of stimulation on SSC variables, we used a linear mixed model with genotype and 
phase as main effects, the genotype $\times$ phase interaction, and individual nested within genotype as a random effect. The dependent variables were the differences between the control and doublet conditions for peak stress, net work, positive work, and negative work per cycle. Because peak stress, net, and negative work changed curvilinearly with phase of stimulation, a quadratic term for phase and the interaction between the quadratic term and genotype was added to the model. A log likelihood ratio test was used to determine the significance of each independent variable. The quadratic term was not significant and therefore not included in the model describing the difference in positive work across genotypes and phase of activation. Because maximum isometric stress was significantly smaller in $m d m$ muscles, the dependent variables were recalculated as the \% difference relative to the control and the analysis was repeated. All variables changed curvilinearly with phase of stimulation, therefore a quadratic term for phase and the interaction between the quadratic term and genotype was also included in the model.

\section{Fiber Bundle Experiments}

We compared four SSC variables across activation levels and between pCa-controlled and BDM-controlled conditions. From the stress-time traces, we calculated the forces at the longest length (peak force) during each SSC. Negative (lengthening phase), positive (shortening phase), and net (total) specific work per cycle were also calculated for each SSC. For clarity, and because trends are similar between SSCs, we only present analysis of SSC 1 in the main text, while data for all SSCs are available in supplemental information (see Supplementary Figure S1). We plotted relative values of force and specific work against activation level.

To assess the effects of condition and force on all four variables, we ran an analysis of covariance (ANCOVA) in JMP. The model included condition ( $\mathrm{pCa}$ vs. $\mathrm{BDM}$ ) as the main effect, activation level as the covariate, the condition by activation level interaction, and individual nested within condition as a random effect. To compare fibers activated with $\mathrm{Ca}^{2+}$ or $\mathrm{Ca}^{2+}$ plus BDM, the ANCOVA analyses were conducted for peak force and work in SSCs from $52 \mathrm{pCa}$ and $76 \mathrm{BDM}$-controlled fibers across activation levels (\% isometric force before the SSCs).

\section{RESULTS}

\section{Whole Muscle Experiments}

In general, when wild type muscles were activated with doublet stimulation during lengthening (phase $<50 \%$ ) force and work increased, and the increase persisted throughout shortening for $100 \mathrm{~ms}$ after stimulation ceased (Figure 3A). When activated during shortening (phase $=50 \%$ ), doublet stimulation resulted in smaller changes in positive and negative work (Figure 3B). Activation at the end of shortening and through the beginning of lengthening (phase $>70 \%$ ) caused a large increase in negative work but small change in positive work (Figure 3C). Across all phases of activation, $m d m$ muscles exhibited little doublet potentiation of force or work (Figures 3D-F).
There was a significantly greater increase in peak stress with doublet stimulation in wild type than in $m d m$ muscles (Figure 4; $F=52.3, p<0.0001$ ). There was also a significant interaction between the quadratic term and genotype, indicating a difference in curvature of the stress-phase relationship between genotypes (Figure 4; $F=13.3, p=0.0004$ ). In wild type soleus, the greatest increase in peak stress occurred at phase $=25 \%$. However, only two muscles were stimulated at this phase, so the mean value may be an over-estimate. For all other phases, at least seven muscles were included in the analysis. In general, the greatest increase in peak stress with doublet stimulation occurred when muscles were activated during lengthening and the smallest increase occurred during shortening. A separate analysis of only $m d m$ data showed no effect of phase $(F=0.2, p=0.65)$ or the quadratic term $(F=0.04, p=0.85)$ on peak stress in $m d m$ muscles (Figure 4). Similarly, the relative increase in peak stress with doublet stimulation was greater in wildtype compared to $m d m$ muscles (Supplementary Figure S1; Supplementary Table S1; $F=23.7$, $p<0.0001)$. However, there was no significant interaction between genotype and the quadratic term, indicating that the relationship between the relative increase in peak stress and phase did not differ between genotypes $(F=0.49, p=0.48)$.

Wild type muscles exhibited a significantly greater increase in net $(F=43.84, p<0.0001)$, negative $(F=14.51, p=0.0007)$, and positive work $(F=50.43, p<0.0001)$ with doublet stimulation compared to $m d m$ muscles (Figure 5). Similar to peak stress, the greatest increase in work occurred when muscles were activated during lengthening and the smallest increase occurred when muscles were activated during shortening. Wild type muscles exhibited a 3 -fold greater increase in negative work with doublet stimulation than $m d m$ muscles, and there was a significant interaction between the quadratic term and genotype on both net $(F=49.81, p<0.0001)$ and negative work $(F=40.87, p<0.0001)$, indicating that the curvature of the relationship between these variables and phase differed between genotypes (Figures 5A,B). Positive work decreased linearly with phase and the slope of this relationship was five times greater in wild type than $m d m$ muscles (Figure 5C; $F=20.88, p<0.0001)$. Relative changes in net, negative, and positive work were similar to absolute differences (Supplementary Figure S2; Supplementary Table S1). During lengthening, the relative increases in both negative and positive work were more than $20 \%$ greater in wild type compared to $m d m$ muscles. Interestingly, at phase $=33 \%$, when wild type muscles were stimulated at the end of lengthening and through the beginning of shortening, there was a more than 2-fold increase in positive work with doublet stimulation, causing net work to shift from negative to positive and to increase by more than 150\% (Supplementary Figure S2).

\section{Permeabilized Fiber Bundle Experiments}

Initial total isometric stress of fiber bundles in $\mathrm{pCa} 4.2$ solution with no BDM was similar (ANOVA; $F=0.07 ; p=0.79$ ) between pCa-controlled $(N=15$; force $=2.05 \pm 0.24 \mathrm{mN}$; PCSA $=0.017 \pm 0.008 \mathrm{~mm}^{2}$; stress $\left.=116.60 \pm 4.2 \mathrm{mN}^{\star} \mathrm{mm}^{2}\right)$ and BDM-controlled fibers $(N=16$; force $=2.24 \pm 0.38$; 
A

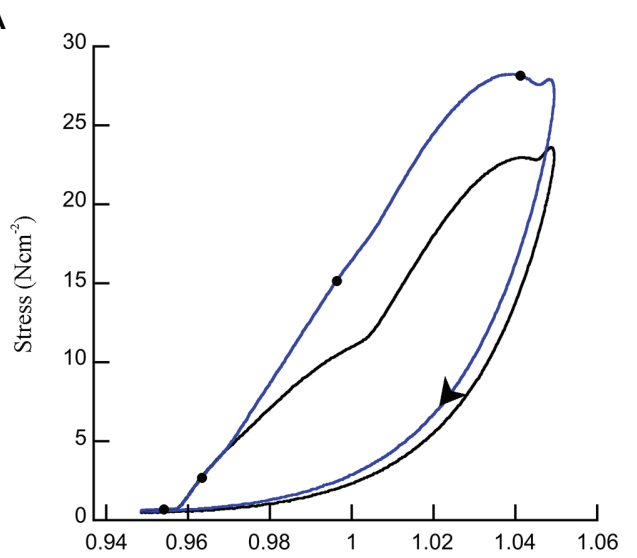

B

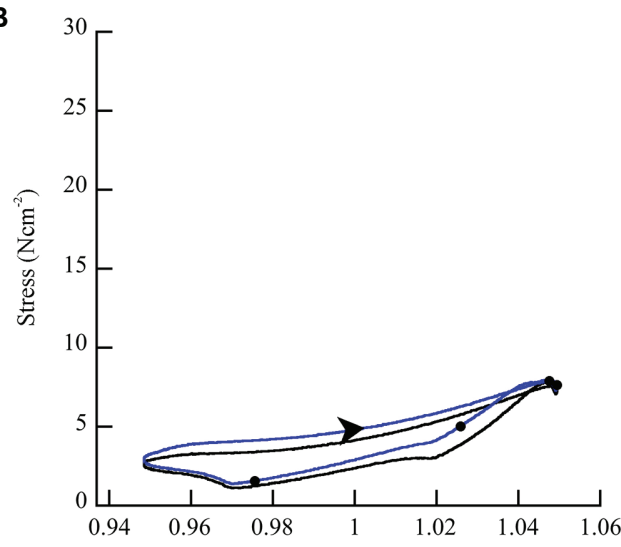

D

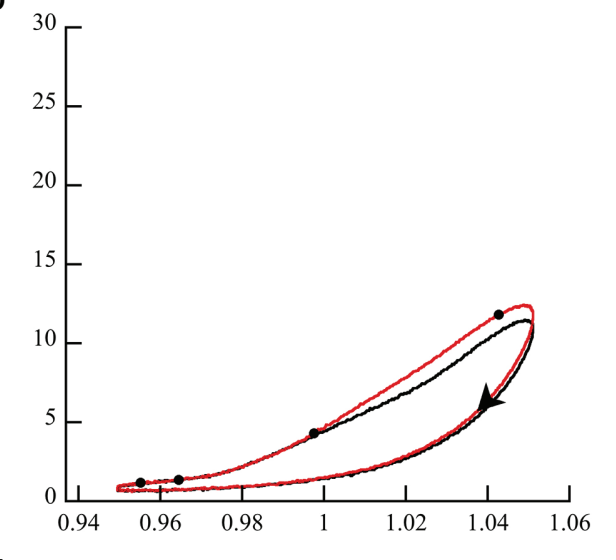

E

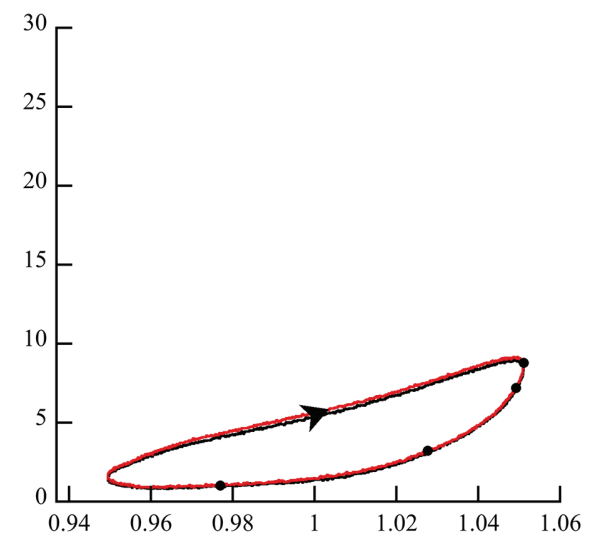

F

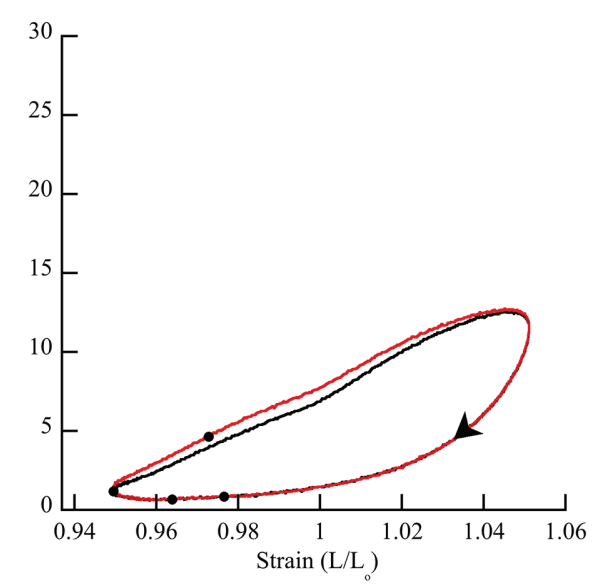

.

30

0.94 c

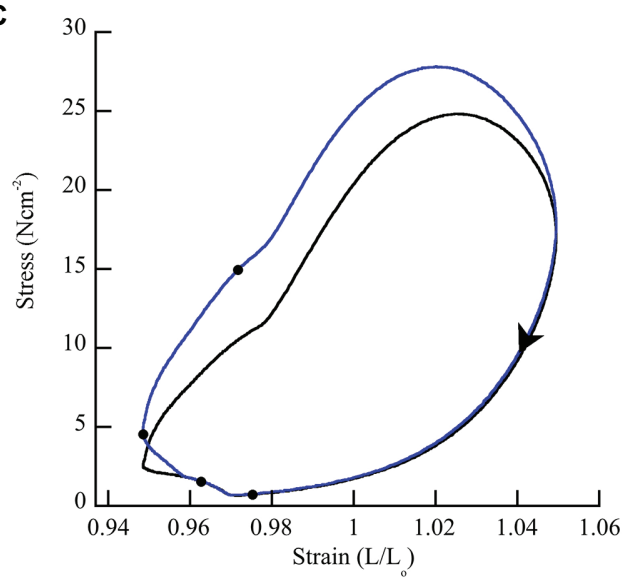

FIGURE 3 | Representative work loops from wild type and $m d m$ soleus muscles stimulated with (blue = wild type; red = $m$ dm) and without (black) a doublet. (A,D) Phase $=8.3 \%$ when the muscle is lengthening. (B,E) Phase $=50 \%$ when the muscle is shortening. $(\mathbf{C}, \mathbf{F})$ Phase $=83.3 \%$ when the muscle is shortening followed by lengthening. The effect of doublet stimulation on work per cycle (area within the work loop) changes with the phase of activation and is greatest when stimulation occurs during lengthening (phase $<50 \%$ ). Black dots indicate individual stimuli. Arrows indicate the direction of a work loop.

PCSA $0.019 \pm 0.012 \mathrm{~mm}^{2} ;$ stress $\left.=119.02 \pm 7.9 \mathrm{mN}^{*} \mathrm{~mm}^{2}\right)$ as expected. Furthermore, the initial isometric stress of relaxed fibers (passive, relaxing solution with no BDM at $2.6 \mu \mathrm{m} \mathrm{SL}$ ) was also similar (ANOVA; $F=0.52 ; p=0.48$ ) between pCa-controlled $\left(12.989 \pm 9.4 \mathrm{mN}^{*} \mathrm{~mm}^{2}\right)$ and BDM-controlled fibers $\left(10.42 \pm 5.9 \mathrm{mN}^{*} \mathrm{~mm}^{2}\right)$.
During the eight SSCs, the first SSC always produced the largest peak force (up to $250 \%$ maximal isometric force), with subsequent SSCs decreasing in force until SSC 5-8 when the force became approximately constant (Supplementary Figure S3). The relationship between peak force during the first SSC vs. activation level differed between $\mathrm{pCa}$ - and $\mathrm{BDM}$-controlled 


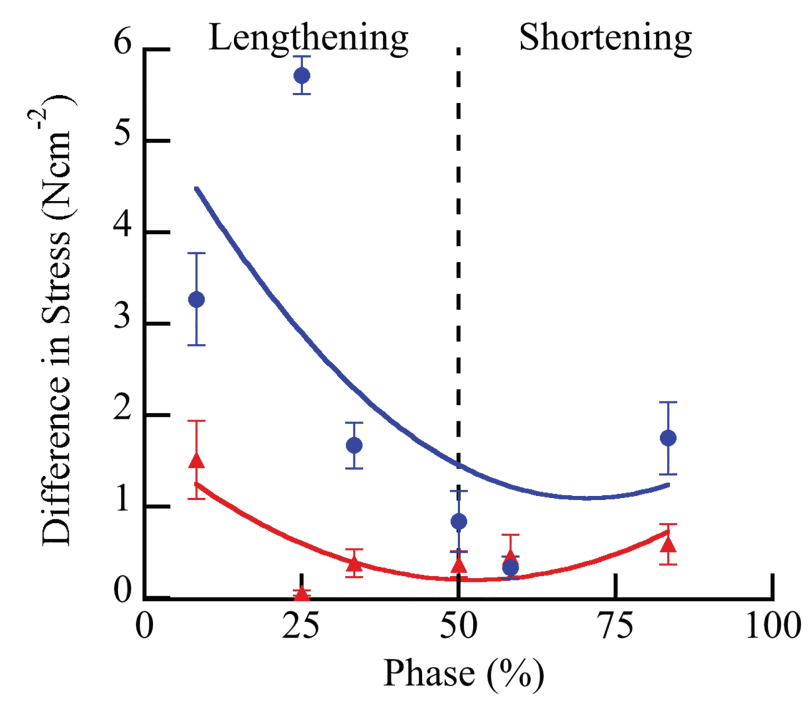

FIGURE 4 | Differences in peak stress with vs. without doublet stimulation across phase of stimulation in wild type (blue) and $m d m$ (red) soleus. Doublet potentiation of peak stress is reduced in $\mathrm{mdm}$ muscles compared to wild type at all phases of activation $(F=52.3, p<0.0001)$. Phase $=0 \%$ indicates a muscle at its shortest length at the onset of lengthening and phase $=50 \%$ indicates a muscle at its longest length just prior to shortening. Data represent means $\pm \mathrm{SE}, N=28$ individuals, 112 trials.

fibers (Figure 6A). ANCOVA showed that peak force increased with increasing activation level for both conditions (activation level covariate $F=680.71, p<0.001$ ). Furthermore, compared to $\mathrm{pCa}$-controlled fibers, the peak force of the BDM-controlled fibers was higher at a given activation level (condition $F=12.59, p=0.0014$ ) and trended toward a steeper slope than in pCa-controlled fibers (interaction $F=3.78, p=0.055$; Figure 6A).

The relationships between specific positive, negative, and net work per cycle vs. activation level also differed between pCa- and BDM-controlled fibers (Figures 6B-D). For all three work variables, there was a significant positive relationship between work and activation level (positive work $F=612.07$, $p<0.001$; negative work $F=1318.01, p<0.001$; net work $F=1399.58, p<0.001)$. The relationship between positive work vs. activation level for BDM-controlled fibers produced a slightly steeper slope than for pCa-controlled fibers (interaction $F=7.53, p=0.0072$ ), and there was no significant difference between intercepts (condition $F=0.41, p=0.53$; Figure 6B). In contrast, the relationship between negative work vs. activation level produced steeper slopes (interaction $F=4.75, p=0.031$ ) and increased intercepts (condition $F=14.89, p<0.001)$ in BDM-controlled fibers compared to pCa-controlled fibers (Figure 6C). Finally, the relationship between net work vs. activation level increased in BDM-controlled fibers compared to pCa-controlled fibers (condition $F=26.78, p<0.001$ ), but with similar slopes (interaction $F=1.90, p=0.1709$; Figure 6D). Taken together, our data indicate that specific negative and net work per cycle is greater in BDM- vs. pCa-controlled fibers.

\section{DISCUSSION}

The focus of our study was to conduct SSC experiments at two different levels of muscle organization to test the hypothesis that $\mathrm{Ca}^{2+}$-activated, non-cross bridge, viscoelastic elements contribute to muscle force and work during dynamic changes in length. We used whole muscle and single fiber preparations to ask whether the traditional sliding filament - swinging cross bridge theory alone can account for muscle force and work during SSCs. Together, both approaches provided support for the hypothesis that a $\mathrm{Ca}^{2+}$-dependent, non-cross bridge element, likely titin, contributes to force and work during SSCs.

\section{Whole Muscle Experiments}

Three observations from whole muscle studies support the existence of non-cross bridge forces in SSCs. (1) In wild type muscles, potentiation of force and work persists for $\sim 100 \mathrm{~ms}$ after doublet stimulation, long after $\sim 25 \mathrm{~ms}\left[\mathrm{Ca}^{2+}\right]$ transients associated with a doublet stimulus have subsided (Bakker et al., 2017). (2) Adding a doublet stimulus to a submaximal pulse train during SSCs increased force and work in a phase-dependent manner, with stimulation during the eccentric phase producing the greatest increase. (3) The response to doublet stimulation was significantly reduced in $m d m$ muscles with a small deletion in N2A titin (Garvey et al., 2002), showing almost no longlasting effects throughout an SSC.

Results from the present study showed that doublet potentiation of force and work persisted throughout an SSC for $100 \mathrm{~ms}$ after stimulation ceased in wild type muscles. This observation corroborates previous findings which showed a rapid increase in force with doublet stimulation that can be sustained for long periods up to $250 \mathrm{~ms}$ under isometric conditions (Burke et al., 1970; Zajac and Young, 1980; Stein and Parmiggiani, 1981; Hennig and Lømo, 1987; Sandercock and Heckman, 1997; Abbate et al., 2002). Recent work by Bakker et al. (2017) showed that doublet stimulation increases intracellular $\left[\mathrm{Ca}^{2+}\right]$ for the first $20-30 \mathrm{~ms}$ following stimulation. They suggested that the $\left[\mathrm{Ca}^{2+}\right]$ transient might saturate the second $\mathrm{Ca}^{2+}$-binding site on $\mathrm{TnC}$ and lead to faster initiation of cross-bridge cycling, although this hypothesis remains to be tested experimentally. Nevertheless, the proposed increase in rate of cross-bridge cycling via saturation of TnC cannot account neither for increased force with doublet stimulation nor for persistence of the potentiated force for up to hundreds of milliseconds (Burke et al., 1976; Sandercock and Heckman, 1997) after the $\left[\mathrm{Ca}^{2+}\right]$ level has returned to baseline levels (Abbate et al., 2002; Cheng et al., 2013; Bakker et al., 2017). Thus, while the rate of cross-bridge cycling may increase with a transient increase in $\left[\mathrm{Ca}^{2+}\right]$ and therefore contribute to the rapid increase in force, neither the time constants of $\mathrm{Ca}^{2+}$ release and re-uptake nor the saturation of $\mathrm{TnC}$ are consistent with the sustained increase in force and work that occurs in response to doublet stimulation.

The increased effect of doublet stimulation during the eccentric phase of an SSC that we observed here matches previous work demonstrating that stretch of a muscle during doublet stimulation 


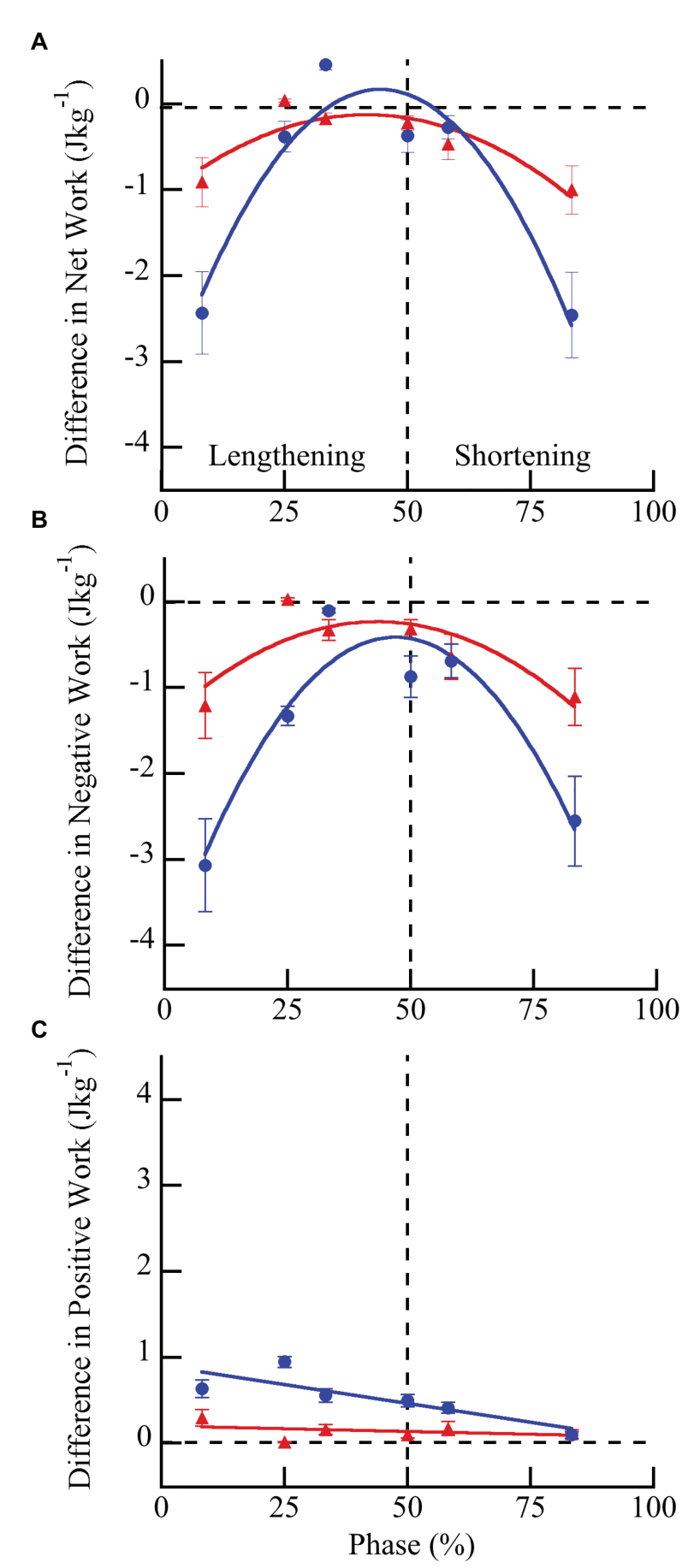

FIGURE 5 | Differences in net (A), negative (B), and positive (C) work per cycle with vs. without doublet stimulation across phase of activation in wild type (blue) and $m d m$ (red) muscles. The greatest increase in work with doublet stimulation occurred when muscles were activated during lengthening and the smallest increase occurred when muscles were activated during shortening. Wild type muscles exhibited a significantly greater increase in net $(F=43.84, p<0.0001)$, negative $(F=14.51$, $p=0.007)$, and positive $(F=50.43, p<0.0001)$ work than $m d m$ muscles across all phases of activation. Phase $=0 \%$ indicates a muscle at its shortest length at the onset of lengthening and phase $=50 \%$ indicates a muscle at its longest length just prior to shortening. Data represent means $\pm \mathrm{SE}, N=28$ individuals, 112 trials.

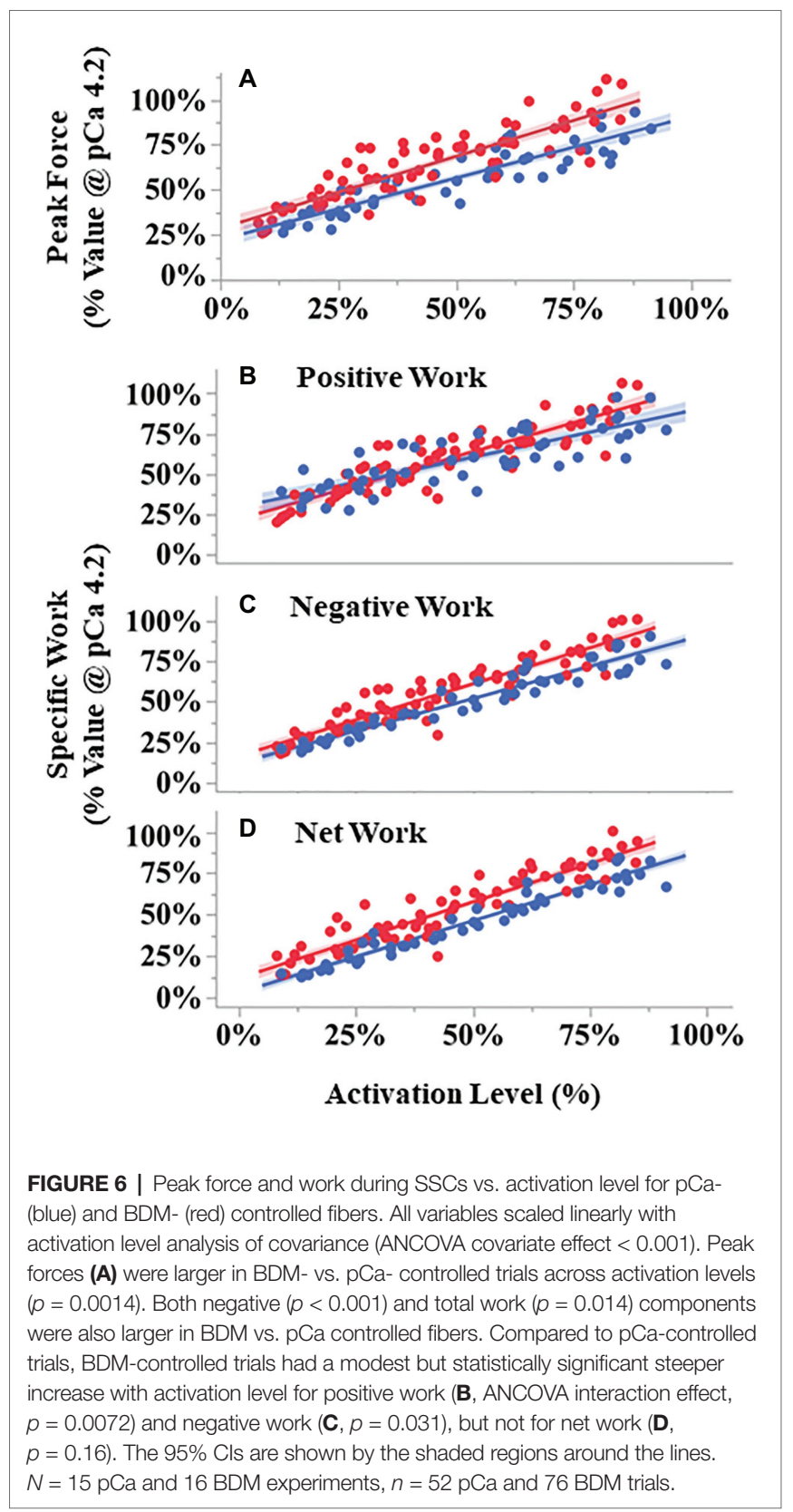

enhances force, whereas shortening abolishes the doublet effect (Sandercock and Heckman, 1997). Isometric studies have also shown that doublet potentiation is greatest at short muscle lengths on the ascending limb of the length-tension relationship and decreases as muscle length increases (Burke et al., 1976; Sandercock and Heckman, 1997). In addition, Stevens (1996) showed that adding a single doublet stimulus to an SSC can increase net work per cycle by more than $50 \%$. The lengthdependence of doublet potentiation is not easily explained by cross bridge models, but instead suggests the engagement of a $\mathrm{Ca}^{2+}$-dependent viscoelastic element (Binder-Macleod and Kesar, 2005) that stores elastic energy during stretch and releases energy during shortening, thereby increasing muscle force and work during eccentric SSCs (Nishikawa et al., 2018). 
Interestingly, results from the present study also showed that doublet potentiation is markedly reduced in $m d m$ muscles, which are characterized by a small deletion in the $\mathrm{N} 2 \mathrm{~A}$ region of titin (Garvey et al., 2002). The difference between wild type and $m d m$ muscles was greatest when the muscles were activated during lengthening, where doublets increased negative, positive, and net work per cycle in wild type but not $m d m$ soleus. These observations are consistent with other studies on $m d m$ muscles, which demonstrated reduced force enhancement during active stretch and reduced force depression with active shortening (Tahir et al., 2020), as well as reduced net work during eccentric SSCs (Hessel and Nishikawa, 2017).

To our knowledge, this study is the first to test the hypothesis that titin contributes to doublet potentiation. Previous studies have suggested a role for elastic elements other than cross bridges (Stein and Parmiggiani, 1981; Sandercock and Heckman, 1997; Binder-Macleod and Kesar, 2005), but the mechanism for doublet potentiation remains unclear. Doublet potentiation is similar to "catch" in molluscan muscles, in that force is maintained for hours at low $\left[\mathrm{Ca}^{2+}\right]$ (Butler and Siegman, 2010). The mechanism for molluscan catch has been shown to involve binding of twitchin, a giant sarcomeric protein related to titin, to thin filaments (Butler and Siegman, 2010), similar to N2A titin-actin binding which is thought to be responsible for titin activation (Dutta et al., 2018; Nishikawa, 2020). The addition of a doublet at the onset of a submaximal stimulus train causes a transient increase in $\left[\mathrm{Ca}^{2+}\right]$, which likely increases in titinactin binding in addition to cross bridge formation. However, once $\left[\mathrm{Ca}^{2+}\right]$ returns to baseline levels between stimuli ( 25 ms), cross bridges release but force remains elevated due to the much slower off rates ( $200 \mathrm{~ms})$ of titin-actin interactions (Dutta et al., 2018). At a cycle frequency of $5 \mathrm{~Hz}$, titin would be expected to remain bound to actin for the entire duration of an SSC. How titin contributes to doublet potentiation of force during isometric contractions remains unclear because activation increases titin stiffness, thus strain is required to increase titin force. Hypotheses including titin winding on actin (Nishikawa et al., 2012) or a "sticky spring" (Rode et al., 2009) could potentially explain how titin contributes to force of isometrically contracting muscle but future work is needed.

This study is also the first to test the hypothesis that titin contributes to the phase-dependence of activation. When activated at its shortest length followed by stretch-shortening, a muscle behaves like a spring in which elastic energy stored during stretch enhances work during shortening. However, when activated at its longest length followed by shortening-stretch, a muscle behaves like a motor which produces positive work (Ahn, 2012). During in vivo movements, the phase-dependence of activation serves the important function of allowing a muscle to function in different ways for different tasks (Dickinson et al., 2000). The observation that the phase dependence of activation is reduced in skeletal muscles from $m d m$ mice suggests a contribution for titin. We suggest that binding of N2A titin to actin at different muscle lengths depending on the phase of activation is a mechanism of phase-dependence. Titin-actin binding is thought to decrease titin free length and increase titin stiffness (Dutta et al., 2018; Nishikawa et al., 2019).
Binding of titin to actin at differing distances from the Z-disk would have the effect of enhancing both the spring-like and motor-like properties of muscles depending on the phase of activation.

\section{Permeabilized Fiber Experiments}

Using permeabilized fiber preparations and the myosin inhibitor $\mathrm{BDM}$, we decoupled the relationships among $\left[\mathrm{Ca}^{2+}\right]$, cross bridge formation, and force production by activating fibers at supramaximal $\left[\mathrm{Ca}^{2+}\right]$ (pCa 4.2) but with reduced cross-bridge forces (Griffiths et al., 2006). We found that peak force, net work, and negative work (Figure 6) were greater in BDM compared to pCa-controlled fiber bundles at a given level of activation.

Early studies found that BDM decreased isometric force more than isometric stiffness. Using permeabilized rabbit psoas fibers, Seow et al. (1997) first reported that treatment with $3 \mathrm{mM}$ BDM increased the stiffness to force ratio by $50 \%$. The increased stiffness to force ratio was thought to be caused by an increase in the number of low-force (i.e., weakly bound) cross bridges that would contribute to stiffness but not active force (Bagni et al., 1992; Seow et al., 1997). However, subsequent analyses (Bagni et al., 1992, 2005; Griffiths et al., 2006) of the effects of BDM on thick and thin filament structure found that the fraction of attached cross bridges is similar between pCa- and BDM-controlled fibers at a given force. Therefore, the BDM-induced increase in the stiffness to force ratio has instead been attributed to the existence of $\mathrm{Ca}^{2+}$-sensitive, non-cross bridge viscoelastic elements (Griffiths et al., 2006; Colombini et al., 2016). BDM has also been used previously to investigate residual force enhancement after isovelocity stretch (Rassier and Herzog, 2005a,b). Using BDM to decrease active force, Rassier and Herzog (2005b) found that a 50\% decrease in active force led to only a $15 \%$ drop in fiber stiffness and a non-statistically significant drop in the absolute residual force enhancement. These observations are also consistent with the existence of a $\mathrm{Ca}^{2+}$-sensitive, non-cross bridge, viscoelastic element that contributes to force enhancement after stretch.

One feature of SSCs is that the magnitude of peak force is positively related to force and work during the subsequent shortening phase (Seiberl et al., 2015b; Fortuna et al., 2017; Hahn and Riedel, 2018; Fukutani and Herzog, 2020a,b). A recent study (Fukutani and Herzog, 2020b) used permeabilized rabbit fibers to compare SSC mechanics between maximally activated fibers at $\mathrm{pCa}=4.2$ with or without $10 \mathrm{mM}$ BDM, reducing the activation level by $\sim 50 \%$. The results showed that positive work produced during the shortening phase of SSCs was relatively larger in BDM-treated fibers, increasing from $169 \%$ of the isovelocity shortening work in $0 \mathrm{mM}$ $\mathrm{BDM}$ to $205 \%$ in $10 \mathrm{mM}$ BDM. In our study, we tested the effect of BDM on positive work during shortening across a range of activation levels from 15 to $100 \%$ maximum activation. We found that compared to $\mathrm{pCa}$-controlled fibers, BDM treatment increased relative positive work only at high activation levels ( $>50 \%$ of the maximal isometric force). At low activation levels ( $<25 \%$ of maximal isometric force), 
positive work of BDM-treated fibers was slightly less than for pCa-controlled fibers (Figure 6B).

An often neglected feature of SSCs is the history-dependent property that peak force (Supplementary Figure S1) is greater during the first repetition in a series of multiple SSCs (see our Figure 2; Campbell and Moss, 2002). This property, called thixotropy (see review by Lakie and Campbell, 2019), is enhanced at lower activation levels and leads to changes in fiber stiffness over time during multiple SSCs. In a series of two consecutive SSCs in single fibers, Campbell and Moss (2002) found that fibers were more compliant during the second stretch phase than the first. However, if a delay was introduced between the two SSCs, then the fibers recovered a portion of their initial stiffness for the second SSC. For delays $>1 \mathrm{~s}$, fiber stiffness became identical between the two SSCs (Campbell and Moss, 2002). They also found that fiber stiffness during the shortening phase was less dependent on length history than during the lengthening phase. The time-dependent thixotropy of fibers during SSCs was not readily predicted by cross bridge models without the addition of a $\mathrm{Ca}^{2+}$-dependent parallel elastic element (Campbell and Moss, 2002).

While it is difficult to separate the contributions of cross bridge and non-cross bridge elements to force production during isometric contraction, Pinniger et al. (2006) conducted a clever set of experiments and used a cross bridge model to distinguish the relative contributions of cross bridge and non-cross bridge elastic elements to muscle force during stretch. They concluded that, after a small stretch of $\sim 1.25-1.5 \% \mathrm{~L}_{0}$, the cross bridge contribution to force enhancement reaches a maximum value, as new detachment/attachment equilibrium is reached. During length changes $>1.5 \% \mathrm{~L}_{0}$, force enhancement must be provided by non-cross bridge elements. Thus, the relative contribution of non-cross bridge elements increases with increasing stretch amplitude. During the SSC experiments reported here, we used strain amplitudes of $10 \%$ in whole muscles and $15 \% \mathrm{~L}_{0}$ in fiber preparations. By extrapolating from Pinniger et al. (2006), the force contribution of non-cross bridge elements is expected to be $\sim 60$ and $\sim 70 \%$ of the total force rise for whole muscles and permeabilized fibers, respectively. However, these calculations are based on maximally activated fibers, where the number of cross bridges is maximized. The calculation therefore overestimates the contribution of cross bridges for the submaximal activation levels used in our SSC experiments. For example, at an activation level of 50\%, the contribution of cross bridges to peak force would be reduced by half, suggesting that cross bridges might contribute as little as 15 or $20 \%$ to peak force in SSCs for permeabilized fibers or whole muscles, respectively.

\section{Titin as a Tunable Viscoelastic Element in Muscle Sarcomeres}

Over the past 20 years, mounting evidence has suggested the existence of a $\mathrm{Ca}^{2+}$-sensitive, non-cross bridge, viscoelastic element in muscle sarcomeres. This element is engaged in the earliest stages of muscle activation at $\left[\mathrm{Ca}^{2+}\right]$ levels below those required to activate thin filaments and permit cross bridge interactions (Bagni et al., 1994, 2002; Colombini et al., 2016). Single myofibril studies also demonstrate that the increase in stiffness with activation following active stretch persists for up to several minutes following deactivation (Joumaa et al., 2007, 2008). Recent estimates suggests that the $\mathrm{Ca}^{2+}$-sensitive non-cross bridge stiffness is 100 times greater than the passive stiffness in isometrically contracting single fibers (Powers et al., 2019). In their study, Powers et al. (2019) used high-frequency length oscillations $(4 \mathrm{kHz})$ in isometrically contracting single muscle fibers to measure fiber stiffness, and found that the I-band stiffness increased by two orders of magnitude in isometrically contracting fibers compared to the passive stiffness of the same fibers at a sarcomere length of $2.7 \mu \mathrm{m} \mathrm{SL}$.

Accumulating evidence further suggests that the giant sarcomeric titin protein is a $\mathrm{Ca}^{2+}$-sensitive, non-cross bridge, viscoelastic element, which functions as a tunable spring in active muscle (Leonard and Herzog, 2010; Nishikawa et al., 2012; Herzog, 2019; Nishikawa, 2020). Numerous recent studies support the hypothesis that $\mathrm{N} 2 \mathrm{~A}$ titin is a signaling hub and mechanical linker that binds to actin in the presence of $\mathrm{Ca}^{2+}$ (Nishikawa et al., 2020), thereby decreasing titin's free length and increasing its stiffness (Dutta et al., 2018; Nishikawa et al., 2019, 2020; Nishikawa, 2020). Single molecule force spectroscopy experiments demonstrate that $\mathrm{N} 2 \mathrm{~A}$-actin interactions are highly stable in the presence of $\mathrm{Ca}^{2+}(\mathrm{pCa}<5.0)$, and the off-rate is more than three times slower $\left(4\right.$ v. $16 \mathrm{~s}^{-1}$ ) when $\mathrm{Ca}^{2+}$ is present (Dutta et al., 2018). Binding of N2A titin to actin brings the titin molecule closer to actin, which may increase the likelihood of hydrostatic interactions between PEVK titin and actin as well. PEVK interactions with actin resist stretch long after $\mathrm{Ca}^{2+}$ has decreased (Bianco et al., 2007).

The $m d m$ mouse model is unique in exhibiting a small deletion in the N2A region of titin (Garvey et al., 2002). This region appears to be critical for mediating the $\mathrm{Ca}^{2+}$-dependent increase in titin stiffness that occurs upon muscle activation (Nishikawa, 2020; Nishikawa et al., 2020). Numerous studies demonstrate that active stiffness is reduced in muscles from $m d m$ mice (Powers et al., 2016; Monroy et al., 2017). These muscles also exhibit reduced force enhancement following active stretch and reduced force depression following active shortening (Tahir et al., 2020), suggesting a role for titin in these historydependent properties.

\section{Limitations}

As is common practice, experiments were conducted at room temperature $\left(25\right.$ and $21^{\circ} \mathrm{C}$ for whole muscle and fiber experiments, respectively) rather than body temperature $\left(37^{\circ} \mathrm{C}\right)$ to reduce fatigue and fiber degradation (Hakim et al., 2013). Although muscle mechanics are altered by temperature (Stephenson and Williams, 1981, 1985; James et al., 2015; Ranatunga, 2018; Caremani et al., 2021), the effect is expected to be relatively small $(\sim 10 \%)$.

2,3-butanedione monoxime is only one way to inhibit cross bridge interactions, with other approaches including the use of different inhibitors such as N-benzyl-p-toluene sulphonamide (BTS; Pinniger et al., 2005), or chemical 
removal of troponin C (Joumaa et al., 2008). Because each method has its own limitations, it is important to repeat these fiber experiments with other cross-bridge inhibitors to compare with data from this study.

\section{Conclusion}

The results from both whole muscle and permeabilized fibers in SSC experiments suggest the existence of a tunable viscoelastic element that enhances muscle force and stores strain energy during active stretch, and recovers stored energy during subsequent shortening to increase positive and net work per cycle. A tunable viscoelastic element such as titin can account for: (1) the persistence of force at low $\left[\mathrm{Ca}^{2+}\right]$ with doublet potentiation; the phase- and length- and phase-dependence of doublet potentiation observed in whole wild type muscles and the absence of these effects in $m d m$ muscles; and lastly, the increased peak force and work per cycle in both whole muscles and permeabilized fibers in SSC experiments. While it has been suggested that other non-cross bridge elements, such as nebulin (Kawai et al., 2018), microtubules (Kerr et al., 2015), and myosin binding protein C (Harris, 2021), may increase muscle stiffness during activation, no studies have demonstrated definitive mechanisms that could help to explain the increase in muscle force and work during dynamic length changes. So far, titin is the only known non-cross bridge viscoelastic element in muscle sarcomeres that has been shown to interact with $\mathrm{Ca}^{2+}$ and actin.

By combining SSC experiments in whole muscles and permeabilized fibers, we gain a better understanding of the $\mathrm{Ca}^{2+}$-dependent viscoelastic elements that contribute to the mechanical properties exhibited by active muscles during dynamic changes in length. Whole muscle studies have the advantage of simulating in vivo muscle activation and length changes, whereas in fiber preparations, we can quantify the relative contributions of cross bridge and non-cross bridge elements to dynamic force during SSCs. Both preparations showed an increase in peak force and work per cycle with increasing activation (adding a doublet in whole muscle experiments and decreasing $\mathrm{pCa}$ in permeabilized fibers), which was enhanced during lengthening contractions. It is important to point out that phase dependence could only be measured in whole muscle studies since muscles were activated with a duty cycle of 0.5 , whereas fibers were activated with a duty cycle of $100 \%$ throughout the entire SSC protocol. Thus, work loops from fiber preparations more closely resembled eccentric work loops of whole muscles. Form these studies, it appears that perturbing activation in SSCs provides an experimental paradigm for quantifying the contributions of calcium-sensitive non-cross

\section{REFERENCES}

Abbate, F., Bruton, J. D., De Haan, A., and Westerblad, H. (2002). Prolonged force increase following a high-frequency burst is not due to a sustained elevation of $\left[\mathrm{Ca}^{2+}\right]$ i. Am. J. Phys. Cell Physiol. 283, C42-C47. doi: 10.1152/ ajpcell.00416.2001

Abbott, B. C., and Aubert, X. M. (1952). The force exerted by active striated muscle during and after change of length. J. Physiol. 117, 77-86. bridge viscoelastic elements to muscle force during dynamic length changes.

\section{DATA AVAILABILITY STATEMENT}

The raw data supporting the conclusions of this article will be made available by the authors, without undue reservation.

\section{ETHICS STATEMENT}

The animal study was reviewed and approved by the Institutional Animal Care and Use Committees of Northern Arizona University (NAU IACUC Protocol \#18-002), the Claremont Colleges (CC IACUC Protocol \#017-003), and the University Clinic Muenster (LANUV NRW, 81-02.04.2019.A472).

\section{AUTHOR CONTRIBUTIONS}

JM and AH contributed equally to this work and were responsible for data collection and analysis. JM, $\mathrm{AH}$, and $\mathrm{KN}$ all contributed to the conception and design of the experiments and interpretation of the data. All authors contributed to the article and approved the submitted version.

\section{FUNDING}

The authors gratefully acknowledge financial support from the National Science Foundation (IOS-1731917 awarded to JM, IOS-2016049 awarded to KN).

\section{ACKNOWLEDGMENTS}

Dr. WA Linke (University of Muenster) provided mouse muscles and research facilities for the fiber study. D. Enright assisted in data collection from whole muscles. A. Good provided critical text editing.

\section{SUPPLEMENTARY MATERIAL}

The Supplementary Material for this article can be found online at: https://www.frontiersin.org/articles/10.3389/fphys.2021.648019/ full\#supplementary-material

Ahn, A. N. (2012). How muscles function-the work loop technique. J. Exp Biol. 215, 1051-1052. doi: 10.1242/jeb.062752

Askew, G. N., and Marsh, R. L. (1997). The effects of length trajectory on the mechanical power output of mouse skeletal muscles. J. Exp. Biol. 200, 3119-3131.

Askew, G. N., and Marsh, R. L. (1998). Optimal shortening velocity (V/Vmax) of skeletal muscle during cyclical contractions: length-force effects and velocity-dependent activation and deactivation. J. Exp. Biol. 201, 1527-1540. 
Bagni, M. A., Cecchi, G., Colombini, B., and Colomo, F. (2002). A non-crossbridge stiffness in activated frog muscle fibers. Biophys. J. 82, 3118-3127. doi: 10.1016/S0006-3495(02)75653-1

Bagni, M. A., Cecchi, G., Colomo, F., and Garzella, P. (1992). Effects of 2,3-butanedione monoxime on the crossbridge kinetics in frog single muscle fibres. J. Muscle Res. Cell Motil. 13, 516-522. doi: 10.1007/BF01737994

Bagni, M. A., Cecchi, G., Colomo, F., and Garzella, P. (1994). Development of stiffness precedes cross-bridge attachment during the early tension rise in single frog muscle fibres. J. Physiol. 481, 273-278. doi: 10.1113/jphysiol.1994. sp020437

Bagni, M. A., Colombini, B., Colomo, F., Berlinguer Palmini, R., and Cecchi, G. (2005). Non cross-bridge stiffness in skeletal muscle fibres at rest and during activity. Adv. Exp. Med. Biol. 565, 141-154. doi: 10.1007/0-387-24990-7_11

Bakker, A. J., Cully, T. R., Wingate, C. D., Barclay, C. J., and Launikonis, B. S. (2017). Doublet stimulation increases $\mathrm{Ca}^{2+}$ binding to troponin $\mathrm{C}$ to ensure rapid force development in skeletal muscle. J. Gen. Physiol. 149, 323-334. doi: 10.1085/jgp.201611727

Bang, M. L., Centner, T., Fornoff, F., Geach, A. J., Gotthardt, M., McNabb, M., et al. (2001). The complete gene sequence of titin, expression of an unusual approximately $700-\mathrm{kDa}$ titin isoform, and its interaction with obscurin identify a novel Z-line to I-band linking system. Circ. Res. 89, 1065-1072. doi: 10.1161/hh2301.100981

Bianco, P., Nagy, A., Kengyel, A., Szatmári, D., Mártonfalvi, Z., Huber, T., et al. (2007). Interaction forces between F-actin and titin PEVK domain measured with optical tweezers. Biophys. J. 93, 2102-2109. doi: 10.1529/ biophysj.107.106153

Binder-Macleod, S., and Kesar, T. (2005). Catchlike property of skeletal muscle: recent findings and clinical implications. Muscle Nerve 31, 681-693. doi: 10.1002/mus.20290

Burke, R. E., Rudomin, P., and Zajac, F. E. (1970). Catch property in single mammalian motor units. Science 168, 122-124. doi: 10.1126/ science.168.3927.122

Burke, R. E., Rudomin, P., and Zajac, F. E. (1976). The effect of activation history on tension production by individual muscle units. Brain Res. 109, 515-529. doi: 10.1016/0006-8993(76)90031-7

Butler, T. M., and Siegman, M. J. (2010). Mechanism of catch force: tethering of thick and thin filaments by twitchin. J. Biomed. Biotechnol. 2010:725207. doi: 10.1155/2010/725207

Campbell, K. S., and Moss, R. L. (2002). History-dependent mechanical properties of permeabilized rat soleus muscle fibers. Biophys. J. 82, 929-943. doi: 10.1016/S0006-3495(02)75454-4

Caremani, M., Fusi, L., Linari, M., Reconditi, M., Piazzesi, G., Irving, T. C., et al. (2021). Dependence of thick filament structure in relaxed mammalian skeletal muscle on temperature and interfilament spacing. J. Gen. Physiol. 153:e202012713. doi: 10.1085/jgp.202012713

Chen, J., Hahn, D., and Power, G. A. (2019). Shortening-induced residual force depression in humans. J. Appl. Physiol. 126, 1066-1073. doi: 10.1152/ japplphysiol.00931.2018

Cheng, A. J., Place, N., Bruton, J. D., Holmberg, H.-C., and Westerblad, H. (2013). Doublet discharge stimulation increases sarcoplasmic reticulum $\mathrm{Ca}^{2+}$ release and improves performance during fatiguing contractions in mouse muscle fibres. J. Physiol. 591, 3739-3748. doi: 10.1113/jphysiol.2013. 257188

Colombini, B., Nocella, M., and Bagni, M. A. (2016). Non-crossbridge stiffness in active muscle fibres. J. Exp. Biol. 219, 153-160. doi: 10.1242/jeb.124370

Daley, M. A., and Biewener, A. A. (2011). Leg muscles that mediate stability: mechanics and control of two distal extensor muscles during obstacle negotiation in the Guinea fowl. Philos. Trans. R. Soc. Lond. Ser. B Biol. Sci. 366, 1580-1591. doi: 10.1098/rstb.2010.0338

Dick, T. J. M., Biewener, A. A., and Wakeling, J. M. (2017). Comparison of human gastrocnemius forces predicted by hill-type muscle models and estimated from ultrasound images. J. Exp. Biol. 220, 1643-1653. doi: 10.1242/ jeb. 154807

Dickinson, M. H., Farley, C. T., Full, R. J., Koehl, M. A., Kram, R., and Lehman, S. (2000). How animals move: an integrative view. Science 288, 100-106. doi: 10.1126/science.288.5463.100

Dutta, S., Tsiros, C., Sundar, S. L., Athar, H., Moore, J., Nelson, B., et al. (2018). Calcium increases titin N2A binding to F-actin and regulated thin filaments. Sci. Rep. 8:14575. doi: 10.1038/s41598-018-32952-8
Edman, K. A. (1975). Mechanical deactivation induced by active shortening in isolated muscle fibres of the frog. J. Physiol. 246, 255-275. doi: 10.1113/ jphysiol.1975.sp010889

Edman, K. A., Elzinga, G., and Noble, M. I. (1982). Residual force enhancement after stretch of contracting frog single muscle fibers. J. Gen. Physiol. 80, 769-784. doi: 10.1085/jgp.80.5.769

Fortuna, R., Groeber, M., Seiberl, W., Power, G. A., and Herzog, W. (2017). Shortening-induced force depression is modulated in a time- and speeddependent manner following a stretch-shortening cycle. Phys. Rep. 5:e13279. doi: 10.14814 /phy2.13279

Fox, J., and Weisberg, S. (2010). An R companion to applied regression. 2nd Edn. Thousand Oaks, Calif: SAGE Publications, Inc.

Fukutani, A., and Herzog, W. (2020a). Differences in stretch-shortening cycle and residual force enhancement between muscles. J. Biomech. 112:110040. doi: 10.1016/j.jbiomech.2020.110040

Fukutani, A., and Herzog, W. (2020b). The stretch-shortening cycle effect is prominent in the inhibited force state. J. Biomech. 115:110136. doi: 10.1016/j. jbiomech.2020.110136

Garvey, S. M., Rajan, C., Lerner, A. P., Frankel, W. N., and Cox, G. A. (2002). The muscular dystrophy with myositis (mdm) mouse mutation disrupts a skeletal muscle-specific domain of titin. Genomics 79, 146-149. doi: 10.1006/ geno.2002.6685

Gordon, A. M., Huxley, A. F., and Julian, F. J. (1966). The variation in isometric tension with sarcomere length in vertebrate muscle fibres. J. Physiol. 184, 170-192. doi: 10.1113/jphysiol.1966.sp007909

Granzier, H. L., and Irving, T. C. (1995). Passive tension in cardiac muscle: contribution of collagen, titin, microtubules, and intermediate filaments. Biophys. J. 68, 1027-1044. doi: 10.1016/S0006-3495(95)80278-X

Griffiths, P. J., Bagni, M. A., Colombini, B., Amenitsch, H., Bernstorff, S., Funari, S., et al. (2006). Effects of the number of actin-bound S1 and axial force on X-ray patterns of intact skeletal muscle. Biophys. J. 90, 975-984. doi: 10.1529/biophysj.105.068619

Hahn, D., and Riedel, T. N. (2018). Residual force enhancement contributes to increased performance during stretch-shortening cycles of human plantar flexor muscles in vivo. J. Biomech. 77, 190-193. doi: 10.1016/j. jbiomech.2018.06.003

Hakim, C. H., Wasala, N. B., and Duan, D. (2013). Evaluation of muscle function of the extensor digitorum longus muscle ex vivo and tibialis anterior muscle in situ in mice. J. Vis. Exp. 50183. doi: 10.3791/50183

Harris, S. P. (2021). Making waves: a proposed new role for myosin-binding protein $\mathrm{C}$ in regulating oscillatory contractions in vertebrate striated muscle. J. Gen. Physiol. 153:e202012729. doi: 10.1085/jgp.202012729

Heidlauf, T., Klotz, T., Rode, C., Siebert, T., and Röhrle, O. (2017). A continuummechanical skeletal muscle model including actin-titin interaction predicts stable contractions on the descending limb of the force-length relation. PLoS Comput. Biol. 13:e1005773. doi: 10.1371/journal.pcbi.1005773

Hennig, R., and Lømo, T. (1987). Gradation of force output in normal fast and slow muscles of the rat. Acta Physiol. Scand. 130, 133-142. doi: 10.1111/ j.1748-1716.1987.tb08119.x

Herzog, W. (2018). The multiple roles of titin in muscle contraction and force production. Biophys. Rev. 10, 1187-1199. doi: 10.1007/s12551-0170395-y

Herzog, W. (2019). The problem with skeletal muscle series elasticity. BMC Biomed. Eng. 1:28. doi: 10.1186/s42490-019-0031-y

Hessel, A. L., Joumaa, V., Eck, S., Herzog, W., and Nishikawa, K. C. (2019). Optimal length, calcium sensitivity and twitch characteristics of skeletal muscles from mdm mice with a deletion in N2A titin. J. Exp. Biol. 222:jeb200840. doi: $10.1242 /$ jeb. 200840

Hessel, A. L., and Nishikawa, K. C. (2017). Effects of a titin mutation on negative work during stretch-shortening cycles in skeletal muscles. J. Exp. Biol. 220, 4177-4185. doi: 10.1242/jeb.163204

Horowits, R., Kempner, E. S., Bisher, M. E., and Podolsky, R. J. (1986). A physiological role for titin and nebulin in skeletal muscle. Nature 323, 160-164. doi: 10.1038/323160a0

Huxley, H. E. (1957). The double array of filaments in cross-striated muscle. J. Biophys. Biochem. Cytol. 3, 631-648. doi: 10.1083/jcb.3.5.631

Huxley, H., and Hanson, J. (1954). Changes in the cross-striations of muscle during contraction and stretch and their structural interpretation. Nature 173, 973-976. doi: 10.1038/173973a0 
Huxley, A. F., and Niedergerke, R. (1954). Structural changes in muscle during contraction; interference microscopy of living muscle fibres. Nature 173, 971-973. doi: 10.1038/173971a0

James, R. S., Altringham, J. D., and Goldspink, D. F. (1995). The mechanical properties of fast and slow skeletal muscles of the mouse in relation to their locomotory function. J. Exp. Biol. 198, 491-502.

James, R. S., Tallis, J., and Angilletta, M. J. (2015). Regional thermal specialisation in a mammal: temperature affects power output of core muscle more than that of peripheral muscle in adult mice (Mus musculus). J. Comp. Physiol. B, Biochem. Syst. Environ. Physiol. 185, 135-142. doi: 10.1007/s00360-014-0872-6

Joumaa, V., Rassier, D. E., Leonard, T. R., and Herzog, W. (2007). Passive force enhancement in single myofibrils. Pflugers Arch. 455, 367-371. doi: $10.1007 / \mathrm{s} 00424-007-0287-2$

Joumaa, V., Rassier, D. E., Leonard, T. R., and Herzog, W. (2008). The origin of passive force enhancement in skeletal muscle. Am. J. Phys. Cell Physiol. 294, C74-C78. doi: 10.1152/ajpcell.00218.2007

Kawai, M., Karam, T. S., Kolb, J., Wang, L., and Granzier, H. L. (2018). Nebulin increases thin filament stiffness and force per cross-bridge in slow-twitch soleus muscle fibers. J. Gen. Physiol. 150, 1510-1522. doi: 10.1085/jgp.201812104

Kerr, J. P., Robison, P., Shi, G., Bogush, A. I., Kempema, A. M., Hexum, J. K., et al. (2015). Detyrosinated microtubules modulate mechanotransduction in heart and skeletal muscle. Nat. Commun. 6:8526. doi: 10.1038/ncomms9526

Kuhn, T. S. (1962). The structure of scientific revolutions. Chicago, USA: University of Chicago Press.

Lakie, M., and Campbell, K. S. (2019). Muscle thixotropy-where are we now? J. Appl. Physiol. 126, 1790-1799. doi: 10.1152/japplphysiol.00788.2018

Lee, S. S. M., Arnold, A. S., de Boef Miara, M., Biewener, A. A., and Wakeling, J. M. (2013). Accuracy of gastrocnemius muscles forces in walking and running goats predicted by one-element and two-element hill-type models. J. Biomech. 46, 2288-2295. doi: 10.1016/j.jbiomech.2013.06.001

Leonard, T. R., and Herzog, W. (2010). Regulation of muscle force in the absence of actin-myosin-based cross-bridge interaction. Am. J. Phys. Cell Physiol. 299, C14-C20. doi: 10.1152/ajpcell.00049.2010

Li, Y., Hessel, A. L., Unger, A., Ing, D., Recker, J., Koser, F., et al. (2020). Graded titin cleavage progressively reduces tension and uncovers the source of A-band stability in contracting muscle. elife 9:e64107. doi: 10.7554/eLife.64107

Linari, M., Woledge, R. C., and Curtin, N. A. (2003). Energy storage during stretch of active single fibres from frog skeletal muscle. J. Physiol. 548, 461-474. doi: 10.1113/jphysiol.2002.032185

Linke, W. A. (2018). Titin gene and protein functions in passive and active muscle. Annu. Rev. Physiol. 80, 389-411. doi: 10.1146/annurevphysiol-021317-121234

Linke, W. A., Ivemeyer, M., Mundel, P., Stockmeier, M. R., and Kolmerer, B. (1998a). Nature of PEVK-titin elasticity in skeletal muscle. Proc. Natl. Acad. Sci. U. S. A. 95, 8052-8057. doi: 10.1073/pnas.95.14.8052

Linke, W. A., Stockmeier, M. R., Ivemeyer, M., Hosser, H., and Mundel, P. (1998b). Characterizing titin's I-band Ig domain region as an entropic spring. J. Cell Sci. 111, 1567-1574.

Lopez, M. A., Pardo, P. S., Cox, G. A., and Boriek, A. M. (2008). Early mechanical dysfunction of the diaphragm in the muscular dystrophy with myositis (Ttnmdm) model. Am. J. Phys. Cell Physiol. 295, C1092-C1102. doi: $10.1093 /$ icb/icr008

Monroy, J. A., Powers, K. A., Gilmore, L. A., Uyeno, T. A., and Nishikawa, K. C. (2012). $\mathrm{Ca}^{2+}$-activation of skeletal muscle: not just the thin filament? Integr. Comp. Biol. 52:E94.

Monroy, J. A., Powers, K. A., Gilmore, L. A., Uyeno, T., and Nishikawa, K. C. (2017). Effects of activation on the elastic properties of intact soleus muscles with a deletion in titin. J. Exp. Biol. 220, 828-836. doi: 10.1242/jeb.139717

Nishikawa, K. (2016). Eccentric contraction: unraveling mechanisms of force enhancement and energy conservation. J. Exp. Biol. 219, 189-196. doi: $10.1242 /$ jeb. 124057

Nishikawa, K. C. (2018). Muscle function from oraganisms to molecules. Integr. Comp. Biol. 58, 194-206. doi: 10.1093/icb/icy023

Nishikawa, K. (2020). Titin: a tunable spring in active muscle. Physiology 35, 209-217. doi: 10.1152/physiol.00036.2019

Nishikawa, K., Dutta, S., DuVall, M., Nelson, B., Gage, M. J., and Monroy, J. A. (2019). Calcium-dependent titin-thin filament interactions in muscle: observations and theory. J. Muscle Res. Cell Motil. 41, 125-139. doi: 10.1007/ s10974-019-09540-y
Nishikawa, K., Lindstedt, S. L., Hessel, A., and Mishra, D. (2020). N2A Titin: signaling hub and mechanical switch in skeletal muscle. Int. J. Mol. Sci. 21:3974. doi: $10.3390 /$ ijms 21113974

Nishikawa, K. C., Lindstedt, S. L., and LaStayo, P. C. (2018). Basic science and clinical use of eccentric contractions: history and uncertainties. J. Sport Health Sci. 7, 265-274. doi: 10.1016/j.jshs.2018.06.002

Nishikawa, K. C., Monroy, J. A., Powers, K. L., Gilmore, L. A., Uyeno, T. A., and Lindstedt, S. L. (2013). A molecular basis for intrinsic muscle properties: implications for motor control. Adv. Exp. Med. Biol. 782, 111-125. doi: 10.1007/978-1-4614-5465-6_6

Nishikawa, K. C., Monroy, J. A., Uyeno, T. E., Yeo, S. H., Pai, D. K., and Lindstedt, S. L. (2012). Is titin a "winding filament"? A new twist on muscle contraction. Proc. Biol. Sci. 279, 981-990. doi: 10.1098/rspb. 2011.1304

Nocella, M., Cecchi, G., Bagni, M. A., and Colombini, B. (2014). Force enhancement after stretch in mammalian muscle fiber: no evidence of crossbridge involvement. Am. J. Phys. Cell Physiol. 307, C1123-C1129. doi: 10.1152/ ajpcell.00290.2014

Parmiggiani, F., and Stein, R. B. (1981). Nonlinear summation of contractions in cat muscles. II. Later facilitation and stiffness changes. J. Gen. Physiol. 78, 295-311. doi: 10.1085/jgp.78.3.295

Paternoster, F. K., Seiberl, W., Hahn, D., and Schwirtz, A. (2016). Residual force enhancement during multi-joint leg extensions at joint- angle configurations close to natural human motion. J. Biomech. 49, 773-779. doi: 10.1016/j.jbiomech.2016.02.015

Pinniger, G. J., Bruton, J. D., Westerblad, H., and Ranatunga, K. W. (2005). Effects of a myosin-II inhibitor (N-benzyl-p-toluene sulphonamide, BTS) on contractile characteristics of intact fast-twitch mammalian muscle fibres. J. Muscle Res. Cell Motil. 26, 135-141. doi: 10.1007/s10974-005-2679-2

Pinniger, G. J., Ranatunga, K. W., and Offer, G. W. (2006). Crossbridge and non-crossbridge contributions to tension in lengthening rat muscle: forceinduced reversal of the power stroke. J. Physiol. 573, 627-643. doi: 10.1113/ jphysiol.2005.095448

Powers, J. D., Bianco, P., Pertici, I., Reconditi, M., Lombardi, V., and Piazzesi, G. (2019). Contracting striated muscle has a dynamic I-band spring with an undamped stiffness one hundred times larger than the passive stiffness. J. Physiol. 598, 331-345. doi: 10.1113/JP278713

Powers, K., Nishikawa, K., Joumaa, V., and Herzog, W. (2016). Decreased force enhancement in skeletal muscle sarcomeres with a deletion in titin. J. Exp. Biol. 219, 1311-1316. doi: 10.1242/jeb.132027

Ranatunga, K. W. (2018). Temperature effects on force and actin-myosin interaction in muscle: a look back on some experimental findings. Int. J. Mol. Sci. 19:1538. doi: 10.3390/ijms19051538

Rassier, D. E., and Herzog, W. (2005a). Force enhancement and relaxation rates after stretch of activated muscle fibres. Proc. Biol. Sci. 272, 475-480. doi: 10.1098/rspb.2004.2967

Rassier, D. E., and Herzog, W. (2005b). Relationship between force and stiffness in muscle fibers after stretch. J. Appl. Physiol. 99, 1769-1775. doi: 10.1152/ japplphysiol.00010.2005

Rivas-Pardo, J. A., Li, Y., Mártonfalvi, Z., Tapia-Rojo, R., Unger, A., Fernández-Trasancos, Á., et al. (2020). A HaloTag-TEV genetic cassette for mechanical phenotyping of proteins from tissues. Nat. Commun. 11:2060. doi: 10.1038/s41467-020-15465-9

Rode, C., Siebert, T., and Blickhan, R. (2009). Titin-induced force enhancement and force depression: a "sticky-spring" mechanism in muscle contractions? J. Theor. Biol. 259, 350-360. doi: 10.1016/j.jtbi.2009.03.015

Sandercock, T. G., and Heckman, C. J. (1997). Doublet potentiation during eccentric and concentric contractions of cat soleus muscle. J. Appl. Physiol. 82, 1219-1228. doi: 10.1152/jappl.1997.82.4.1219

Seiberl, W., Paternoster, F., Achatz, F., Schwirtz, A., and Hahn, D. (2013). On the relevance of residual force enhancement for everyday human movement. J. Biomech. 46, 1996-2001. doi: 10.1016/j.jbiomech.2013.06.014

Seiberl, W., Power, G. A., and Hahn, D. (2015a). Residual force enhancement in humans: current evidence and unresolved issues. J. Electromyogr. Kinesiol. 25, 571-580. doi: 10.1016/j.jelekin.2015.04.011

Seiberl, W., Power, G. A., Herzog, W., and Hahn, D. (2015b). The stretchshortening cycle (SSC) revisited: residual force enhancement contributes to increased performance during fast SSCs of human $\mathrm{m}$. adductor pollicis. Phys. Rep. 3:e12401. doi: 10.14814/phy2.12401 
Seow, C. Y., Shroff, S. G., and Ford, L. E. (1997). Detachment of low-force bridges contributes to the rapid tension transients of skinned rabbit skeletal muscle fibres. J. Physiol. 501, 149-164. doi: 10.1111/j.1469-7793.1997.149bo.x

Siebert, T., Rode, C., Herzog, W., Till, O., and Blickhan, R. (2008). Nonlinearities make a difference: comparison of two common hill-type models with real muscle. Biol. Cybern. 98, 133-143. doi: 10.1007/s00422-007-0197-6

Stein, R. B., and Parmiggiani, F. (1981). Nonlinear summation of contractions in cat muscles. I. Early depression. J. Gen. Physiol. 78, 277-293. doi: 10.1085/ jgp.78.3.277

Stephenson, D. G., and Williams, D. A. (1981). Calcium-activated force responses in fast- and slow-twitch skinned muscle fibres of the rat at different temperatures. J. Physiol. Lond. 317, 281-302. doi: 10.1113/jphysiol.1981. sp013825

Stephenson, D. G., and Williams, D. A. (1985). Temperature-dependent calcium sensitivity changes in skinned muscle fibres of rat and toad. J. Physiol. 360, 1-12. doi: 10.1113/jphysiol.1985.sp015600

Stevens, E. D. (1996). Effect of phase of stimulation on acute damage caused by eccentric contractions in mouse soleus muscle. J. Appl. Physiol. 80, 1958-1962. doi: 10.1152/jappl.1996.80.6.1958

Tahir, U., Monroy, J. A., Rice, N. A., and Nishikawa, K. C. (2020). Effects of a titin mutation on force enhancement and force depression in mouse soleus muscles. J. Exp. Biol. 223:jeb197038. doi: 10.1242/jeb.197038
Taylor-Burt, K. R., Monroy, J., Pace, C., Lindstedt, S., and Nishikawa, K. C. (2015). Shiver me titin! Elucidating titin's role in shivering thermogenesis. J. Exp. Biol. 218, 694-702. doi: 10.1242/jeb.111849

Tsuchiya, T., and Sugi, H. (1988). Muscle stiffness changes during enhancement and deficit of isometric force in response to slow length changes. Adv. Exp. Med. Biol. 226, 503-511.

Zajac, F. E., and Young, J. L. (1980). Properties of stimulus trains producing maximum tension-time area per pulse from single motor units in medial gastrocnemiu muscle of the cat. J. Neurophysiol. 43, 1206-1220. doi: 10.1152/ jn.1980.43.5.1206

Conflict of Interest: The authors declare that the research was conducted in the absence of any commercial or financial relationships that could be construed as a potential conflict of interest.

Copyright (c) 2021 Hessel, Monroy and Nishikawa. This is an open-access article distributed under the terms of the Creative Commons Attribution License (CC BY). The use, distribution or reproduction in other forums is permitted, provided the original author(s) and the copyright owner(s) are credited and that the original publication in this journal is cited, in accordance with accepted academic practice. No use, distribution or reproduction is permitted which does not comply with these terms. 